\title{
Single Trial EEG Patterns for the Prediction of Individual Differences in Fluid Intelligence
}

\author{
Emad-ul-Haq Qazi ${ }^{1 *}$, Muhammad Hussain ${ }^{1}$, Hatim Aboalsamh ${ }^{1}$, Aamir Saeed Malik ${ }^{2}$, \\ Hafeez Ullah Amin ${ }^{2}$ and Saeed Bamatraf ${ }^{1}$ \\ ${ }^{1}$ Visual Computing Lab, Department of Computer Science, College of Computer and Information Sciences, King Saud \\ University, Riyadh, Saudi Arabia, ${ }^{2}$ Centre for Intelligent Signal and Imaging Research (CISIR), Department of Electrical and \\ Electronic Engineering, Universiti Teknologi PETRONAS, Seri Iskandar, Malaysia
}

Assessing a person's intelligence level is required in many situations, such as career counseling and clinical applications. EEG evoked potentials in oddball task and fluid intelligence score are correlated because both reflect the cognitive processing and attention. A system for prediction of an individual's fluid intelligence level using single trial Electroencephalography (EEG) signals has been proposed. For this purpose, we employed 2D and 3D contents and 34 subjects each for 2D and 3D, which were divided into low-ability $(\llcorner A)$ and high-ability $(H A)$ groups using Raven's Advanced Progressive Matrices (RAPM) test. Using visual oddball cognitive task, neural activity of each group was measured and analyzed over three midline electrodes ( $F z, C z$, and Pz). To predict whether an individual belongs to LA or HA group, features were extracted using wavelet decomposition of EEG signals recorded in visual oddball task and support vector machine (SVM) was used as a classifier. Two different types of Haar wavelet transform based features have been extracted from the band $(0.3$ to $30 \mathrm{~Hz})$ of EEG signals. Statistical wavelet features and wavelet coefficient features from the frequency bands $0.0-1.875 \mathrm{~Hz}$ (delta low) and $1.875-3.75 \mathrm{~Hz}$ (delta high), resulted in the 100 and $98 \%$ prediction accuracies, respectively, both for 2D and 3D contents. The analysis of these frequency bands showed clear difference between LA and HA groups. Further, discriminative values of the features have been validated using statistical significance tests and inter-class and intra-class variation analysis. Also, statistical test showed that there was no effect of 2D and 3D content on the assessment of fluid intelligence level. Comparisons with state-of-the-art techniques showed the superiority of the proposed system.

Keywords: electroencephalography (EEG), fluid intelligence, cognitive task, discrete wavelet transform (DWT), machine learning classifier

\section{INTRODUCTION}

Individual differences are of wide practical importance in educational psychology and provide an opportunity to investigate concepts of cognitive functions (Gray et al., 2003). Fluid intelligence or general fluid intelligence (gf) is a major measurement of individual differences, which reflects the ability of reasoning and solving novel problems, i.e., tasks that cannot be solved as a function of simple memorization. It is also indirectly related to learning, memory retention and recall process 
(Ricardo et al., 2010). Various studies on cognitive tasks have linked the fluid intelligence with human learning ability and capacity (Deary et al., 2007; Van den Bos et al., 2012; Wang et al., 2013), which cannot be assessed subjectively i.e., by mere memorization and answering the questions (Primi et al., 2010). The alternative is to use direct brain activations, for which EEG signals can be used.

Electroencephalography (EEG) is a commonly used neuroimaging technique to analyze neural processing and can be used to assess a subjects' fluid intelligence level. A common approach to study cognitive processes is to use event-related potentials (ERP), a component of EEG. Recent research has shown that ERP exhibited variations while subjects performed various cognitive tasks (Polich, 2007; Ubeyli, 2009; Wronka et al., 2013). ERP represents averaged time locked brain voltage variations in EEG recordings, which are related to cognitive tasks. Due to averaging, ERP can be misleading because it might not reflect the actual brain dynamics of subjects (Gaspar et al., 2011). On the other side, single trial EEG signals provide the information that is not accessible using the conventional analysis of peak amplitudes and latencies of ERP (Quiroga et al., 2007). Single-trial analysis can provide a systematic mapping between (i) brain activity and stimulus information space (Schyns, 2010; Rousselet et al., 2011), (ii) brain activity and subject's behavioral variability (Ratcliff et al., 2009), and (iii) brain activity measured using different imaging techniques, e.g., fMRI and EEG (Goldman et al., 2009; deBettencourt et al., 2011). The focus of most of the previous research was on the classification of EEG signals based on different cognitive tasks and rest condition tasks, i.e., eyes open and eyes closed (baseline tasks), and no one addressed the problem of assessing the intelligence level of individuals.

In order to assess a subject's fluid intelligence level, we employed single trial EEG signals and assumed two fluid intelligence levels, i.e., LA and HA. As such, it was modeled as a two-class classification problem. To model a classification system, we collected EEG signals from 34 subjects while watching 2D and $3 \mathrm{D}$ contents. One pattern recognition system was developed for $2 \mathrm{D}$ and $3 \mathrm{D}$ each. Firstly, the RAPM test was used to divide the subjects into two groups (i.e., LA and HA) based on their intellectual ability. Next, we used the visual oddball cognitive task to measure the neural activity of each group by presenting target and standard stimuli and measured the brain activation as EEG signals from three sites $F z, P z, C z$. After preprocessing the EEG signals, Haar wavelet transform was used to extract the statistical wavelet features (SWF) and wavelet coefficient features (WCF) from the low-frequency bands. Then the state-of-theart classification technique, i.e., SVM with RBF kernel was used for our system to predict whether a subject belongs to LA or HA group. The proposed system gives promising results for the prediction of fluid intelligence level.

The main contributions of this study are (i) a system for the prediction of a subject's fluid intelligence level using single trial EEG signals, (ii) two different feature descriptors based on Haar wavelet transform, which are easy to compute and are effective in discriminating LA and HA groups, (iii) the analysis of low frequency bands showing that there is a clear difference between
EEG signals belonging to LA and HA groups. To validate the discriminative values of the features, we employed the statistical significance test, and inter-class and intra-class variation analysis.

The rest of the paper is organized as follows: In Section Literature Review we present the literature review. Section Materials and Methods describes in detail the proposed method. Experimental results and discussion are given in Section Experimental Results and Discussion, while Section Conclusion concludes the paper.

\section{LITERATURE REVIEW}

The prediction of a subject's fluid intelligence level is a classification problem, which involves extracting discriminatory features from EEG signals and classification. Though, according to our knowledge, no study have been conducted which deal with the prediction of a subject's fluid intelligence level so far, there have been a number of studies on the classification of EEG signals for various similar cognitive tasks. In the following paragraphs, we reviewed those methods, which used wavelet transform (WT), time and frequency-domain techniques for feature extraction (Iscan et al., 2011).

WT, time and frequency-domain techniques have been widely used to extract features from cognitive and rest conditions' tasks. Out of these techniques, WT showed very good performance as compared to other methods due to its compliance with the EEG brain signals which having non-stationary behavior. The WT features which are considered for analysis are statistical features (standard deviations, mean and median) (Yazdani et al., 2009; Garry et al., 2013), wavelet entropy (Rosso et al., 2001) and wavelet coefficients (Orhan et al., 2011). These features have been used for analysis in EEG and clinical applications. The features based on time domain are Lyapunov exponent (Ubeyli, 2010), Hurst component (Acharya et al., 2012), Hjorth parameters, fractal dimension, permutation entropy (Vidaurre et al., 2009), approximate entropy and sample entropy (Richman and Moorman, 2000). Frequency-domain features in various frequency bands are power ratio, EEG absolute power and relative power (Thatcher et al., 2005). The analysis of timefrequency signals include stockwell transform and WT based feature extraction (Hariharan et al., 2014). Guo et al. (2011) utilized the immune feature along with the weighted SVM. The author performed the classification of cognitive tasks and achieved the accuracy between 85.4 and $97.5 \%$. Hariharan et al. (2014) utilized the stockwell transform for the extraction of discriminatory features. They carried out the classification by using the SVM. For this purpose, they used the EEG signals recorded from various cognitive tasks. Accuracy rate of 84.72 to $98.95 \%$ was achieved by the authors during the classification phase. Zhang et al. (2010) used the Fischer's discriminant classifier and high-frequency power for the classification of EEG signals, recorded during the cognitive tasks. They achieved the classification accuracy between 72.4 and $76.4 \%$. Hosni et al. (2007) used the power feature of EEG signals. For classification, they utilized the SVM with a radial basis function (RBF) 
kernel. They achieved the accuracy of $70 \%$ by performing the classification on three cognitive tasks.

Xue et al. (2003) achieved the accuracy of $85.3 \%$ by using the RBF classifier. They used the wavelet packet transform as a feature extractor. In another study, Zhiwei and Minfen (2007) used the wavelet pack entropy as a feature. By applying the SVM classifier, they achieved the accuracy between 87.5 and $93.0 \%$. In this scheme, they showed the discrimination between cognitive and baseline task. In the experiment, five tasks were performed by the database of seven subjects, i.e., (1) eyes open (baseline) task, (2) mental letter composing task, (3) geometric object rotation task, (4) multiplication task and (5) visual counting task. Keirn and Aunon (1990) developed this database at Colorado State University, which was based on simple cognitive tasks consisting of seven subjects only. In some research studies, authors utilized the database of few subjects for the classification purpose. As in Zhiwei and Minfen (2007), authors utilized only the database of two subjects. Similarly, in another research study, Nai-Jen and Palaniappan (2004) used the database of four subjects in their experimentation. Researchers also worked on classification of EEG brain signals, which were recorded during different cognitive tasks, by utilizing different databases developed by authors in their research studies. Lin and Hsieh (2009) achieved the accuracy of $78.31 \%$ by using the neural network classifier. They used the EEG power features for the classification of cognitive tasks. Rodriguez-Bermudez et al. (2013) achieved the accuracy of $67.96-80.71 \%$ by utilizing the wavelet, time and frequency based features. They used the SVM as classifier. In another research study, Karkare et al. (2009) utilized the scaling exponent as a feature. Artificial neural network was utilized as a classifier to classify the two groups. These two groups did the cognitive task of complex nature. They achieved the accuracy of $80 \%$. The above-discussed studies showed the low accuracy of classification. Many of these studies used the non-linear classifiers like artificial neural networks. These classifiers were time consuming in constructing the models for the classification purpose. Jahidin et al. (2014) used the progressive metric test, i.e., Raven as a cognitive task. They obtained the accuracy of $88.89 \%$. They utilized the EEG power and artificial neural network as a feature and classifier, respectively.

The above literature review indicates that wavelet transform is the most effective tool for extracting discriminative features from EEG signals. As such, we felt motivated to employ WT to propose an efficient feature extraction and classification (online and offline) system that can predict the fluid intelligence level of the subjects, whether they belong to HA or LA group by performing the cognitive task using $2 \mathrm{D}$ and $3 \mathrm{D}$ contents.

\section{MATERIALS AND METHODS}

The objective of this study is to develop a system for predicting the fluid intelligence level of a subject, whether he/she belongs to low or high ability group. One system is developed for $2 \mathrm{D}$ and $3 \mathrm{D}$ content each, for assessing the intelligence level.

First, we selected the material, which consisted of 2D and 3D contents and the subjects for experiments to collect the data.
The subjects were divided into LA and HA groups using RAPM test. In most of the applications, initially it is enough to identify weather a subject belongs to low or high ability group (Amin et al., 2015a). Next, EEG signals were recorded by performing the visual oddball cognitive task for neural activity. These EEG signals were preprocessed to remove the artifacts and noisy signals. Then discriminatory features were extracted from the preprocessed signals by using discrete wavelet transform. Finally, the most representative and relevant features were selected, which were input to the classifier to predict the intelligence level. An overview of the system is given in Figure 1.

\section{Experimental Material and Subjects}

For performing experiments, to collect data for each of $2 \mathrm{D}$ and $3 \mathrm{D}$ contents, 34 healthy male subjects were selected to participate in cognitive tasks. They were all healthy students. 31 were right-handed, and the remaining three were left handed students. Their age range was from 20 to 30 years. They were all medically fit and free from neurological disorders and hearing impairments, and were not using any medication. They possessed corrected to normal or normal vision. All subjects were briefed about the experiment. All of them showed their consent and signed the consent form before the test. In visual oddball cognitive task, Target (sphere) and Standard (box) stimuli were shown to the subjects as $2 \mathrm{D}$ and $3 \mathrm{D}$ contents. The Human Research Ethics Committee of the Universiti Sains Malaysia and Ethics Coordination Committee of the Universiti Teknologi PETRONAS approved this research study.

\section{Data Collection Procedure}

Before the start of the experiment, each subject was briefed about the procedure of data collection and the schedule. According to the availability of subjects, the experiment was carried out on the individual basis. Before the experiment, each subject was asked to solve 10 descriptive questions. These questions acted as a pretest for the subjects. They were related to experimental learning content. The purpose of these questions was to control the subject's background knowledge. Ten percent was the exclusion criteria. It means that maximum one right answer was allowed from each subject. If he/she gave more than one correct answer, then he/she was excluded from the test. No subject indicated the past background information about the experimental learning contents used as a part of this analysis. It confirmed the balance between the LA and HA groups. Every subject was briefed on the experimental methodology, and their seating was arranged in partly sound attenuated room. Then all subjects were given the RAPM test to divide into two groups, i.e., LA and HA. The number of subjects in HA and LA groups in 2D case were 17 each, whereas, in 3D case, the number of subjects were 15 in HA group and 19 in LA group. Next, each subject participated in the visual oddball task. During this task, EEG cap was mounted on the subject's head to capture the EEG signals. The duration of the experiment was about $04 \mathrm{~min}$. Each subject was seated about 1.5 meters away from the TV screen. The size of the screen, on which the task was shown, was about 41 inches. E-Prime Professional, version 2.0 (Psychology Software Tools, Inc., Sharpsburg, PA) was utilized for designing and implementation of this task 


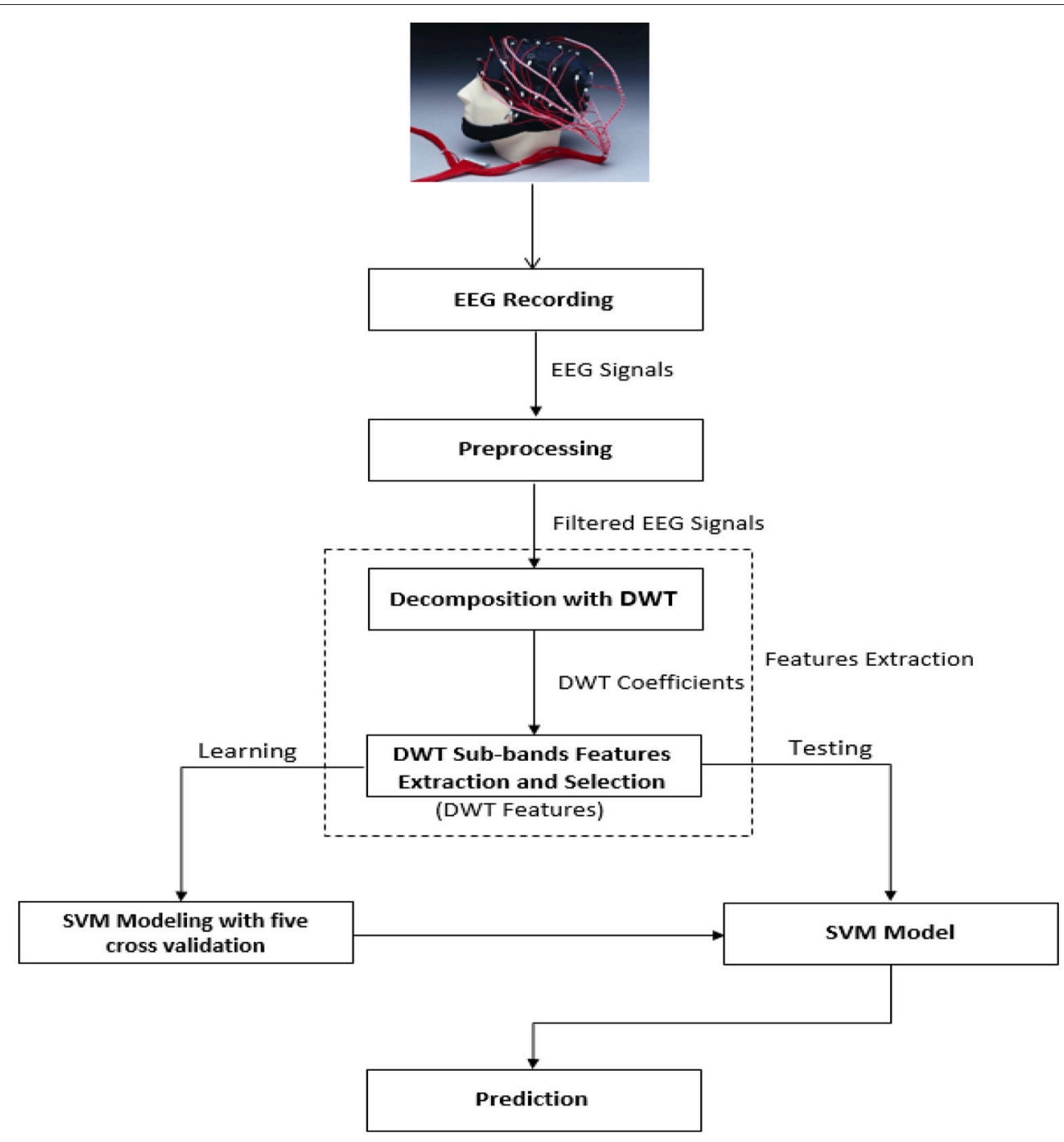

FIGURE 1 | Proposed methodology for feature extraction and classification of EEG signals.

(Schneider et al., 2002). The detail of the above-mentioned cognitive tasks has been discussed in the next sections.

\section{Cognitive Tasks}

Raven's Advanced Progressive Matrices (RAPM) Test

Raven's Advanced Progressive Matrices (RAPM) test (Raven, 2000) is a non-verbal test, which is used to measure the intelligence level of a subject. RAPM measures the two types of fluid cognitive ability, i.e., (i) ability to draw meaning out of confusion, and (ii) ability to recall and reproduce information that has been made explicit and communicated from one to another. It comprises 48 patterns, which are divided into two sets (I and II). Set-I contains 12 patterns, which are used for practice; Set-II contains 36 patterns that are used to assess cognitive ability. Each pattern consists of nine $3 \times 3$-cells, where each cell represents a geometrical shape except the right-bottom cell, which is empty and is to be filled from given eight options. A sample is shown in Figure 2. A subject has to fill the empty cells in 36 patterns, each carrying one score. A score of " 1 " is assigned for each correct answer and a score of " 0 " for an incorrect answer. Total scores range from 0 to 36 . Processing time ranges from $10 \mathrm{~min}$ for Set-I to $40 \mathrm{~min}$ for Set-II (Raven, 2000; Amin et al., 2013). Details of RAPM scores of LA and HA groups for 2D and 3D cases are shown in Table $\mathbf{1}$.

RAPM scores for both the groups were roughly normally distributed as shown in Figures 3, 4 .

No subject was eliminated because each subject's RAPM score was between MEAN - 3SD $(22.85-18.48=4.37)$ and MEAN $+3 \mathrm{SD}(22.85+18.48=41.33)$ in 2D case. Similarly, in 3D case, each subject's RAPM score was between MEAN - 3SD (23.18 $-15.84=7.34)$ and MEAN + 3SD $(23.18+15.84=39.02)$. Moreover, the mean age of the two groups was almost similar. In case of $2 \mathrm{D}$, the mean age of HA group was $23.39(S D= \pm 3.29)$ years and that of LA group was $24.05(S D= \pm 2.25)$ years. In case of 3D, it was $22.81(S D= \pm 2.65)$ years for HA group and 24.24 $(S D= \pm 2.73)$ years for LA group. 


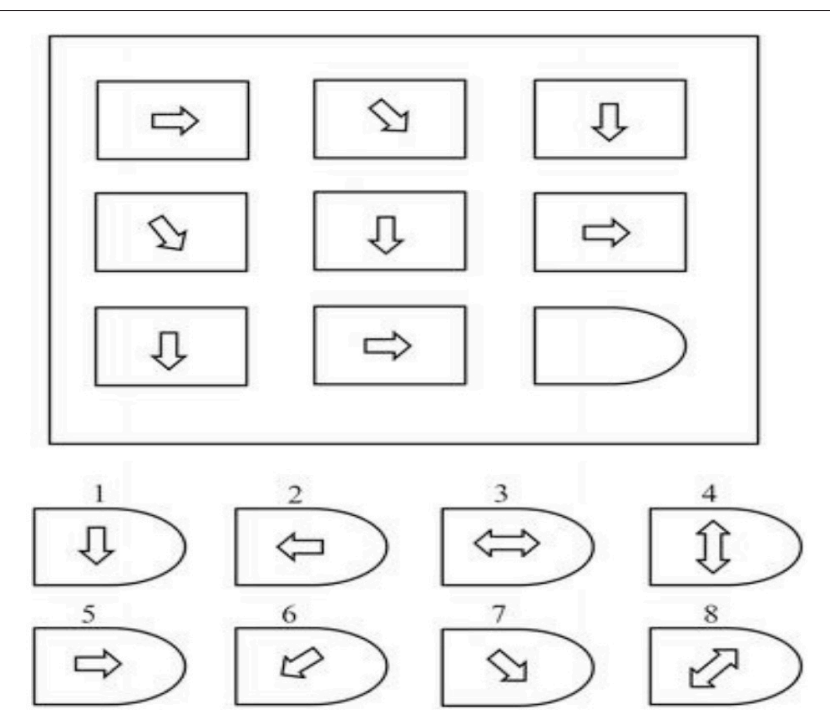

FIGURE 2 | An example of RAPM test (Amin et al., 2015a).

TABLE 1 | Details of RAPM Scores of HA (High Ability) and LA (Low Ability) Groups for 2D and 3D cases.

\begin{tabular}{|c|c|c|c|c|c|c|c|c|}
\hline \multirow{3}{*}{$\begin{array}{l}\text { No. of } \\
\text { subjects }\end{array}$} & \multicolumn{4}{|c|}{ 2D Case } & \multicolumn{4}{|c|}{ 3D Case } \\
\hline & \multicolumn{2}{|c|}{ HA } & \multicolumn{2}{|c|}{ LA } & \multicolumn{2}{|c|}{ HA } & \multicolumn{2}{|c|}{ LA } \\
\hline & $\begin{array}{l}\text { Subject } \\
\text { ID }\end{array}$ & Score & $\begin{array}{l}\text { Subject } \\
\text { ID }\end{array}$ & Score & $\begin{array}{l}\text { Subject } \\
\text { ID }\end{array}$ & Score & $\begin{array}{l}\text { Subject } \\
\text { ID }\end{array}$ & Score \\
\hline 1 & 1 & 27 & 2 & 21 & 1 & 26 & 2 & 22 \\
\hline 2 & 3 & 24 & 4 & 17 & 3 & 24 & 4 & 17 \\
\hline 3 & 8 & 32 & 5 & 13 & 5 & 33 & 8 & 21 \\
\hline 4 & 9 & 29 & 6 & 21 & 6 & 28 & 13 & 23 \\
\hline 5 & 10 & 28 & 7 & 17 & 7 & 24 & 14 & 23 \\
\hline 6 & 11 & 28 & 12 & 22 & 9 & 27 & 15 & 19 \\
\hline 7 & 17 & 27 & 13 & 19 & 10 & 32 & 16 & 16 \\
\hline 8 & 18 & 29 & 14 & 21 & 11 & 34 & 19 & 23 \\
\hline 9 & 19 & 31 & 15 & 12 & 12 & 32 & 20 & 13 \\
\hline 10 & 22 & 30 & 16 & 18 & 17 & 24 & 21 & 17 \\
\hline 11 & 24 & 25 & 20 & 20 & 18 & 24 & 22 & 22 \\
\hline 12 & 26 & 29 & 21 & 22 & 26 & 26 & 23 & 23 \\
\hline 13 & 27 & 24 & 23 & 13 & 30 & 30 & 24 & 21 \\
\hline 14 & 28 & 26 & 25 & 23 & 31 & 25 & 25 & 15 \\
\hline 15 & 32 & 24 & 29 & 6 & 34 & 25 & 27 & 14 \\
\hline 16 & 33 & 28 & 30 & 19 & - & - & 28 & 23 \\
\hline 17 & 34 & 31 & 31 & 21 & - & - & 29 & 19 \\
\hline 18 & - & - & - & - & - & - & 32 & 23 \\
\hline 19 & - & - & - & - & - & - & 33 & 20 \\
\hline
\end{tabular}

Median in 2D case $=23.5$ and $3 D$ case $=23$.

\section{Visual Oddball Task}

The visual oddball task is commonly used for ERP research studies. In this study, visual stimuli were shown to subjects to invoke the neural activities in the attention and cognitive demanding events (Polich, 2007). All selected subjects

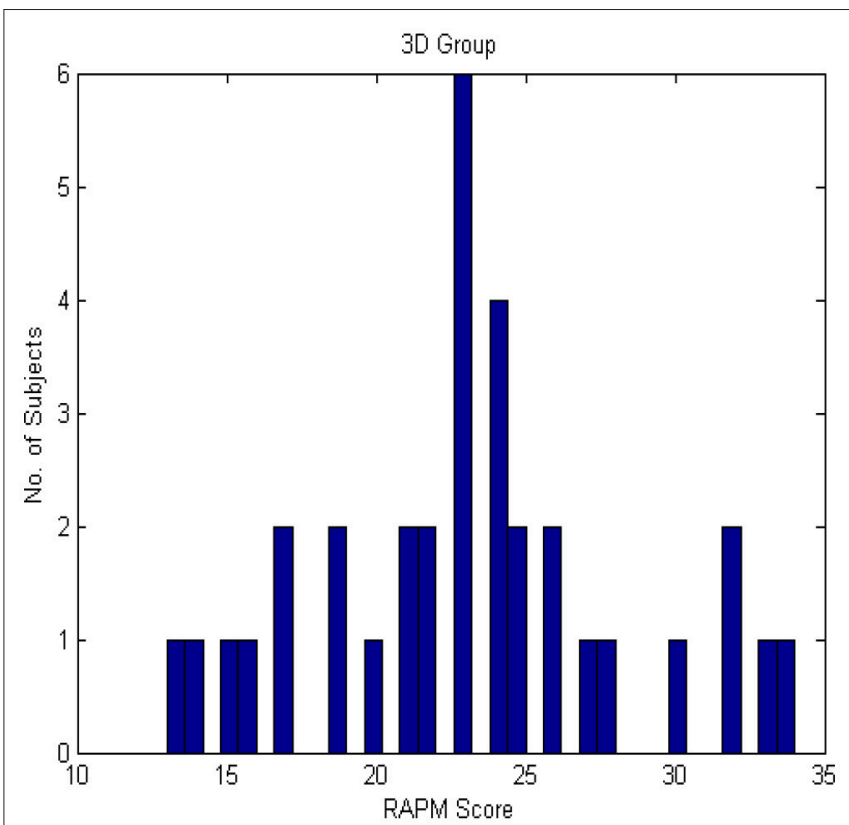

FIGURE 3 | Histogram of RAPM score for 3D group.

participated in the visual oddball task. During visual oddball cognitive task, we used two types of stimuli (standard, which was box large, medium, or small box and target, which was a sphere). Both target and standard stimuli had the same size of $5 \mathrm{~cm}$. Each stimulus appeared on a screen for a duration of $500 \mathrm{~ms}$; there was a pre-stimulus period of $100 \mathrm{~ms}$ before the appearance of each stimulus. The subject was required to press " 0 " when a target shape appeared, and no response was required when standard shapes appeared. Subjects were instructed to respond as quickly as possible to avoid errors. Between the appearances of two stimuli, a black screen was displayed for $1000 \mathrm{~ms}$. There were $30 \%$ target trials and $70 \%$ non-target (standard) trails. As such, out of 135 (total) trials, 40 were target trials, which were used for experiments. The task was performed in accordance with the modification recommended in Huettel and McCarthy (2004).

\section{EEG Recording}

For recording EEG signals from each subject while undergoing visual oddball task, we used the HydroCel Geodesic Sensor Net (Electrical Geodesic Inc., Eugene, OR, USA). It consisted of 128 scalp electrodes as shown in Figure 5. The study by Jongsma et al. (2012) for tracking recall performance and eventrelated potentials (ERPs) across multiple trials in a digit-learning task used midline electrodes $(F z, C z, P z)$. In addition, while studying the (visual or cognitive fatigue) effects of stereoscopic 3D display technology on ERP components, Amin et al. (2015b) also used these midline electrodes. Motivated by these studies, we employed these electrodes to predict the fluid intelligence level of a subject. And our findings indicated that these electrodes are most suitable for this purpose. Therefore, we used only three midline channels to record the EEG signals, i.e., $F z, P z, C z$. 10-20 International System was used for the placement of 128 


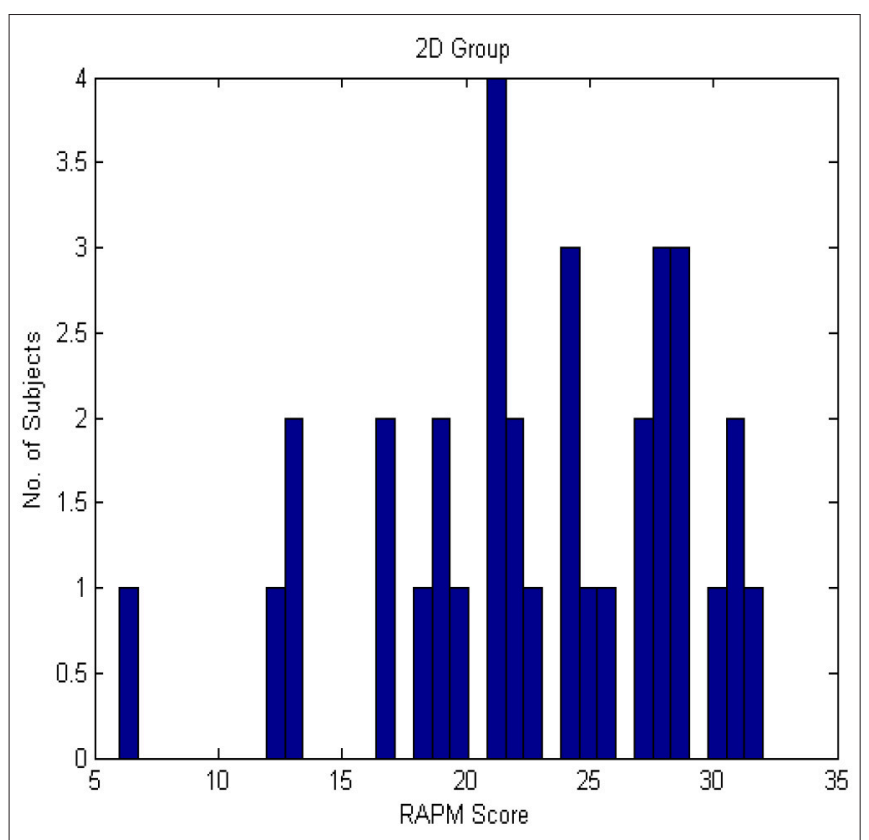

FIGURE 4 | Histogram of RAPM score for 2D group.

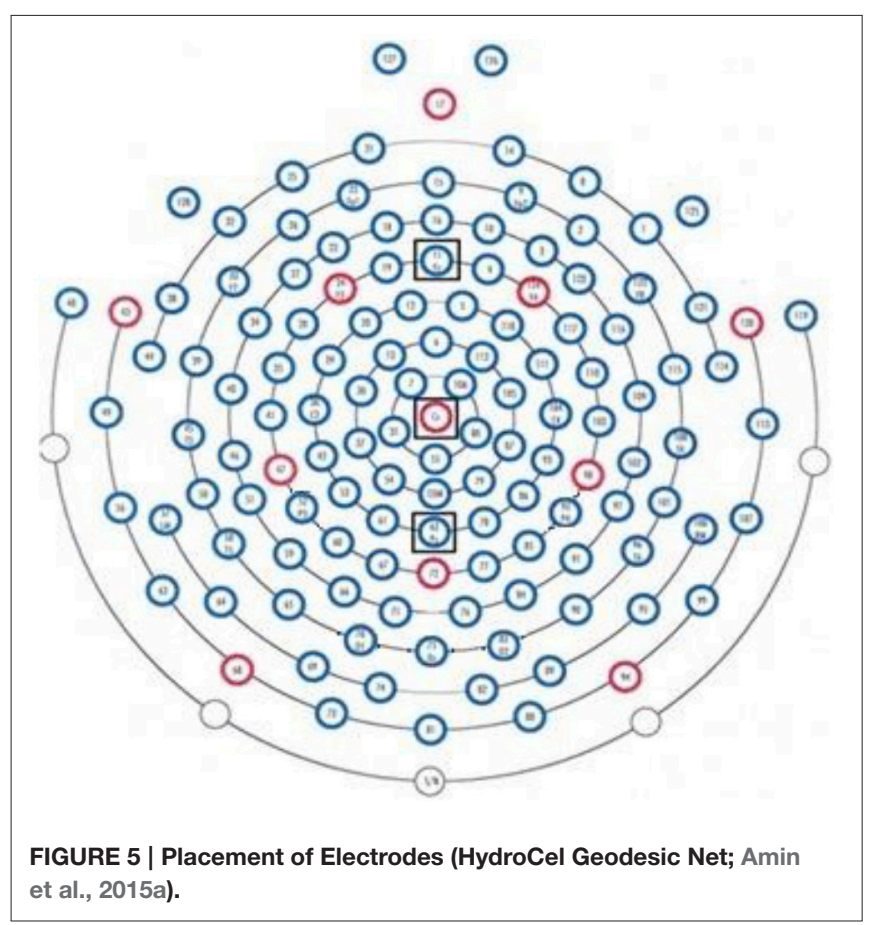

electrodes on the scalp. The $250 \mathrm{~Hz}$ was used as a sampling rate and $50 \mathrm{~K} \Omega$ was utilized as an upper limit of impedance. $C z$ was used as a single vertex electrode and all other electrodes were referenced from it. Amplification of raw signals was carried out from $C z$ with the EGI NetAmps 300 amplifier's band pass filter $(0.1-100 \mathrm{~Hz})$.

From previous studies, it is known that different brain regions may be associated to different brain functions. We used three channels ( $F z, P z, C z)$ for EEG recording due to following reasons (Teplan, 2002):

- $F z$ near intentional and motivational centers,

- $P z$ contribute to activity of perception and differentiation,

- $C z$ location deals with sensory and motor functions.

\section{Data Preprocessing}

The recorded EEG signals involved artifacts. In order to remove the artifacts NetStation v4.5.4 software (Electrical Geodesic, Inc. Eugene, OR, USA) was used to preprocess the EEG signals. First, for removing the muscular artifacts of high frequency and DC components, a band pass filter was utilized (roll off $12 \mathrm{~dB}$ octave, $0.3-30 \mathrm{~Hz}$ ). Next, segmentation of EEG trials related to each subject was performed by using a window of duration $600 \mathrm{mS}$, which contains the baseline, i.e., pre-stimulus period of $100 \mathrm{mS}$ and post stimulus period of $500 \mathrm{mS}$. The trials which involved artifacts like eye movements and eye blinks were rejected, for example, if the amplitude of the EEG signal of any trial was $\pm 90 \mu \mathrm{V}$ then it was rejected. Visual inspection was used for all the trial segments and the contribution of electrodes, which had no contact in the phase of widespread drift (Balas and Koldewyn, 2013), was removed. Spherical spline method (Ferree, 2000) was used to discard a trial if any bad channel was found.

\section{Feature Extraction}

The single trials or epochs belong to two classes, i.e., LA and HA group. After preprocessing, discriminatory features are extracted from EEG signals of each trial. In this section, we proposed two techniques for feature extraction utilizing DWT, commonly used to analyze the biomedical signals based on their time-frequency content (Jahankhani et al., 2006; Orhan et al., 2011). An overview is given in the feature extraction module of Figure 1.

The band passed EEG signal $(0.3-30 \mathrm{~Hz})$ corresponding to one epoch consists of three components corresponding to the channels $F z, P z$, and $C z$, we represent it as follows:

$$
X(t)=[f(t), p(t), c(t)]
$$

where $f(t), p(t)$ and $c(t)$ correspond to $F z, P z$ and $C z$ channels.

First, DWT with Haar wavelet was used to decompose each of $f(t), p(t)$ and $c(t)$ into sub-bands. This resulted in approximate and detail coefficients as shown in Figure 6.

\section{Selection of Wavelet Decomposition Level}

The maximum number of decomposition levels depends on the required frequency components of the signals (Akay, 1997; Adeli et al., 2003; Subasi, 2007; Ocak, 2008). We decomposed a signal upto level 4.

In previous studies (Dimitriadis et al., 2010; Harper et al., 2014), delta bands have been associated with attention and cognitive tasks. The studies involving the event-related potential (ERP) revealed the relationship of delta band with cognitive processes i.e., P300 component is associated with cognitive process (Ergen et al., 2008; Harper et al., 2014). Gennady (2012) reviewed the relationship of delta band with cognitive processes and confirmed this association. These studies reported significant increase in delta power during cognitive tasks. The range of 


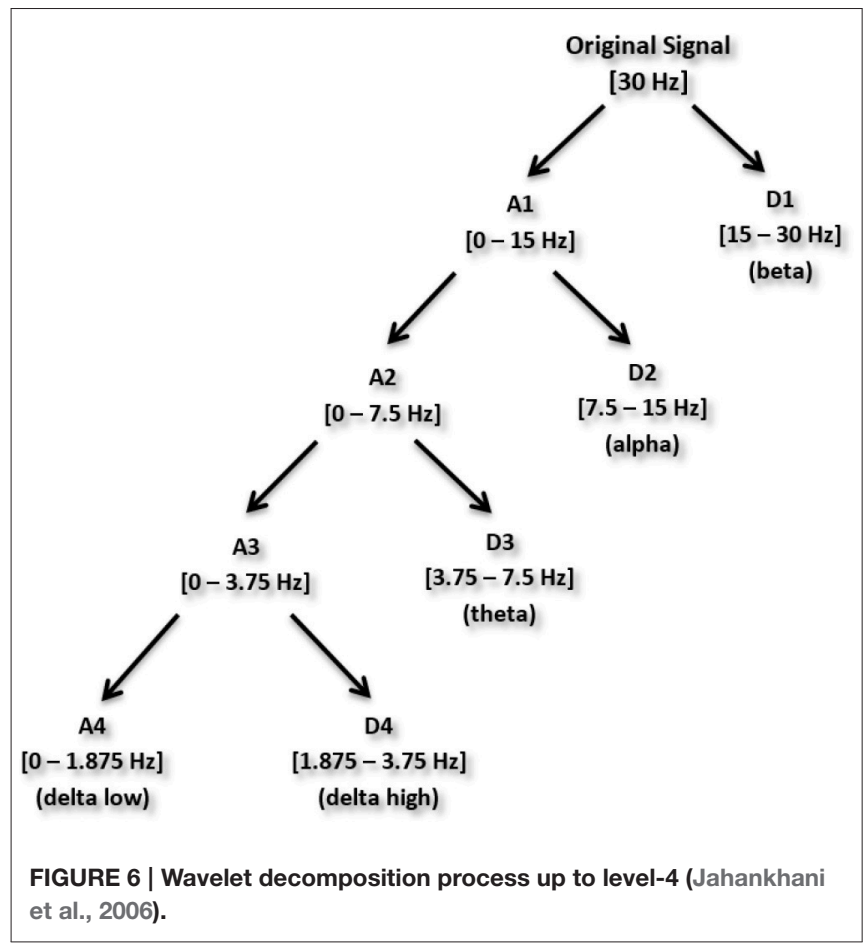

delta band is from 0 to $3.75 \mathrm{~Hz}$ and the decomposition shown in Figure 6 indicates that $A_{4}^{s}$ and $D_{4}^{s}$ bands at decomposition level 4 represent delta band. As such, we decomposed the signal upto level 4. The $A_{4}^{s}, D_{4}^{s}, D_{3}^{s}, D_{2}^{s}$ and $D_{1}^{s}$ components of a subject's EEG signal of single trial at $\mathrm{Fz}$ channel are shown in Figure 7.

The high-pass filter $g(n)$ is the discrete mother wavelet, and the low-pass filter $h(n)$ is its mirror version (Soltani, 2002; Orhan et al., 2011). The approximate and detail coefficients are computed by convolving each component of $X(t)$ with the translates and dilates $\phi_{j, k}(t), \psi_{j, k}(t)$ of scaling function $\phi(t)$ and wavelet function $\psi(t)$, respectively, defined as follows:

$$
\begin{aligned}
& \phi_{j, k}(t)=2^{\frac{j}{2}} h\left(2^{j} n-k\right) \\
& \psi_{j, k}(t)=2^{\frac{j}{2}} g\left(2^{j} n-k\right)
\end{aligned}
$$

where $t=0,1,2, \ldots . ., M-1$ is sampling time point, $j=1,2, \ldots \ldots, J$ is the level of decomposition, $k=$ $0,1,2, \ldots \ldots, 2^{j}-1$ is the translation and $J=4$ (Gonzalez and Woods, 2002). The detail $\left(D_{j}\right)$ and approximation $\left(A_{j}\right)$ coefficients at the $j^{\text {th }}$ level are computed as follows:

$$
\begin{aligned}
& D_{j, k}=X(t)^{*} \psi_{j, k}(t) \\
& A_{j, k}=X(t)^{*} \phi_{j, k}(t)
\end{aligned}
$$

where $j=1,2,3,4$ with $J=4$ and $k=0,1,2, \ldots ., 2^{j}-1$.

The energy of $A_{j}$ and $D_{j}$ at each decomposition level $j=$ $1,2,3,4$ is computed as follows:

$$
\begin{aligned}
& E_{A_{j}}=\sum_{i=1}^{k_{j}}\left|A_{j, i}\right|^{2} \\
& E_{D_{j}}=\sum_{i=1}^{k_{j}}\left|D_{j, i}\right|^{2}
\end{aligned}
$$



FIGURE 7 | Decomposition of single trial EEG signal of one subject at Fz channel.

where $j=1,2,3,4$ and $k_{j}=2^{j}-1$,

$$
E_{\text {total }}=E_{A_{J}}+\sum_{j=1}^{J} E_{D_{j}}
$$

Relative energy of each band is:

$$
E_{r}=\frac{E_{j}}{E_{\text {total }}}
$$

where $E_{j}=E_{D_{j}}, j=1,2,3,4$.

After decomposing EEG signal, we extracted the features using two different approaches:

- Statistical Wavelet Features (SWF),

- Wavelet Coefficients Features (WCF).

\section{Statistical Wavelet Features}

After the decomposition (upto level $J=4$ ) of each component $s=\{f(t), p(t), c(t)\}$ of $X(t)$, we obtained approximation coefficients $A_{4}^{s}$ and detail coefficients $D_{4}^{s}, D_{3}^{s}, D_{2}^{s}$ and $D_{1}^{s}$, the total number of these coefficients was equal to the total number of samples in $s$. From each of $A_{4}^{s}, D_{4}^{s}, D_{3}^{s}, D_{2}^{s}$ and $D_{1}^{s}$, we computed six statistical features [i.e., relative energy $\left(E_{r}\right)$, mean ( $m$ ) standard deviation (std), kurtosis ( $k$ ), skewness $(s k)$ and entropy $(e)]$ and formed a feature vector of dimension 90 for three components. When we divided each component upto level 4 , we obtained five bands. Then we extracted six features six from each band. It means that there are 30 features for each component of $X(t)$. As there are three components, $s=\{f(t), p(t), c(t)\}$, so, there are total number of 90 features for five bands and three components. These features form the feature vector, which represents a trial. These feature vectors were selected from all the trials of the subjects. Therefore, by utilizing all the three components, feature vector is:

$$
V=\left[E_{r}, m, s t d, k, s k, e\right]
$$


We tested the effect of $A_{4}^{s}, D_{4}^{s}, D_{3}^{s}, D_{2}^{s}$ and $D_{1}^{s}$ individually for each component $s=\{f(t), p(t), c(t)\}$ of $X(t)$ and with different combinations to find out which combination gives the best discrimination. Also, we tested different combinations of channels $f(t), p(t)$ and $c(t)$ and different combinations of statistical features.

\section{Wavelet Coefficients Features}

In WCF, we used the approximation coefficients $A_{4}^{s}$ and detail coefficients $D_{4}^{s}, D_{3}^{s}, D_{2}^{s}$ and $D_{1}^{s}$ that were extracted from each component $s=\{f(t), p(t), c(t)\}$ of $X(t)$. SWF features were computed using statistics of these coefficients. Now the question is whether these coefficients have discriminatory information and can be used directly as features. For this purpose, we used the WT coefficients directly as features.

After the decomposition (upto level $J=4$ ), we obtained 153 coefficients from $A_{4}^{s}, D_{4}^{s}, D_{3}^{s}, D_{2}^{s}$ and $D_{1}^{s}$ for each component $s=\{f(t), p(t), c(t)\}$ of $X(t)$. To select which coefficients are discriminative, we proposed two techniques:

- With thresholding (WT),

- Without thresholding (WoT).

Intuitively, the small coefficients indicate that the small amount of corresponding frequencies is present in the signal and such frequencies may be unimportant for discrimination. In WT technique, we threshold the extracted coefficients to eliminate the coefficients having values below a threshold $\mathcal{E}$. We tested four different values of threshold, i.e., $\mathcal{E}=$ $0.5,0.1,0.01,0.001$ to see the effect of thresholding. For thresholding, we used the algorithm presented in Stollnitz et al. (1995).

In WoT technique, we used all coefficients without eliminating the small ones. The purpose was to check whether eliminating the small coefficients affects the prediction accuracy.

\section{Feature Selection}

Some SWF features may not be relevant and discriminative. Redundant features not only cause the curse of the dimensionality problem but also affect the prediction accuracy. In this section, we discussed the selection of discriminatory SWF features. The purpose is to select relevant features and decrease the dimension of the feature space by removing redundant features. For features selection, we used Area under ROC curve that is a robust and efficient method for the selection of relevant features. This method selects only the discriminatory features by calculating their importance (Theodoridis and Koutroumbas, 2009).

In addition, we tested experimentally all the possible combinations of extracted features. We also examined different combinations of three channels and sub-bands $\left(A_{4}\right.$ and $D_{1}$ to $\left.D_{4}\right)$. By using these two methods, we selected two features, i.e., $E_{r}$ and $s t d$, which gave the higher prediction accuracy. Table 2 represents $f(t)$ channel's sub-band percentage $E_{r}$ and its frequency range of a subject against the single trial.
TABLE $2 \mid D_{1}$ to $D_{4}$ represents the detail coefficients from level 1 to 4 and $A_{4}$ represents approximation coefficient at level 4.

\begin{tabular}{llccc}
\hline Levels & $\begin{array}{l}\text { Wavelet } \\
\text { coefficients }\end{array}$ & $\begin{array}{l}\text { Frequency } \\
\text { bands }(\mathbf{H z})\end{array}$ & \multicolumn{2}{l}{ Wavelet relative energy $\left(\mathrm{E}_{\mathbf{r}}\right) \mathbf{( \% )}$} \\
\cline { 5 - 5 } & & $\mathbf{2 D}$ & 3D \\
\hline 1 & D1 & $15-30$ & 0.64 & 1.13 \\
2 & D2 & $7.5-15$ & 2.43 & 4.1 \\
3 & D3 & $3.75-7.5$ & 3.62 & 9.3 \\
4 & D4 & $1.875-3.75$ & 18.57 & 8.38 \\
4 & A4 & $0-1.875$ & 74.74 & 77.09 \\
\hline
\end{tabular}

\section{Classification}

To classify the LA and HA groups is a two-class classification problem. Support Vector Machines (SVM) showed excellent performance in many two-class problems, and as such we used it in our system. It is based on large margin theory and uses the decision function with maximum margin. Though SVM is linear classifier but with kernel trick, it successfully handles non-linearly separable data. Commonly used kernel function is radial basis function (RBF), which gives good performance in a large number of applications, so we used soft SVM with RBF kernel, which has two parameters, i.e., soft margin parameter $C$ and RBF kernel parameter $(\gamma)$. These parameters were tuned using grid search method (Hussain et al., 2011) and five-fold cross-validation.

\section{EXPERIMENTAL RESULTS AND DISCUSSION}

To discuss the results of the proposed scheme, firstly, we give the evaluation protocol, then we discuss the selection of frequency bands using WCF features. After that, results with SWF features have been presented and discussed. Finally, the discrimination of SWF features has been analyzed using statistical significance tests and inter-class and intra-class variations.

\section{Evaluation Protocol}

To evaluate the classification system, we used tenfold cross validation technique where the given data was divided into 10folds. Each fold was held out in turn and remaining 9-folds were used to train and tune the system. After training and tuning the system, the left-over fold was used as an independent set to test the performance of the system. This process was repeated for each fold and average performance values were calculated. The main advantage of this technique was that the system was tested under various samples of data.

To tune the parameters $C$ and $\gamma$ of SVM, we used $25 \%$ of the training data, five cross validation and grid search.

For evaluating the performance of the system, we employed the commonly used measures, such as accuracy, precision, sensitivity and specificity, which are defined below: 


$$
\begin{aligned}
\text { Accuracy } & =\frac{\begin{array}{c}
\text { Total No. of Correctly } \\
\text { Classified Instances }
\end{array}}{\text { Total No. of Instances }} \times 100 \\
\text { Precision } & =\frac{\mathrm{TP}}{\mathrm{TP}+\mathrm{FP}} \times 100 \\
\text { Sensitivity } & =\frac{\mathrm{TP}}{\mathrm{TP}+\mathrm{FN}} \times 100 \\
\text { Specificity } & =\frac{\mathrm{TN}}{\mathrm{TN}+\mathrm{FP}} \times 100
\end{aligned}
$$

Where,

TP: True Positive: A subject predicted with LA when he/she actually has LA,

TN: True Negative: A subject predicted with HA when he/she actually has HA,

FP: False Positive: A subject predicted with LA when he/she actually has HA, and,

FN: False Negative: A subject predicted with HA when he/she actually has LA.

In $2 \mathrm{D}$ case, the total number of trials (epochs) for HA group was of 551, whereas this number was 482 for LA group. Similarly, in $3 \mathrm{D}$ case, the total number of trials was 433 for HA group and 529 for LA group.

\section{Selection of Wavelet Bands}

After decomposition of $X(t)$, we checked $A_{4}^{s}, D_{4}^{s}, D_{3}^{s}, D_{2}^{s}$, and $D_{1}^{s}$ by plotting different trials of LA and HA group, and observe the maximum discrimination in $A_{4}^{s}$ and $D_{4}^{s}$ which represents the delta low and delta high band. On the other hand, we also performed experiments on all decomposition levels with SWF and WCF features, and found that it is in accordance with our above observation that $A_{4}^{s}$ and $D_{4}^{s}$ have discriminative information. We checked all the bands, and best performance is coming from delta band. The prediction accuracy for $A_{4}^{s}$ and $D_{4}^{s}$, i.e., $100 \%$ were higher than the $D_{3}^{s}, D_{2}^{s}$ and $D_{1}^{s}$ components. The accuracy rate for $D_{3}^{s}, D_{2}^{s}$ and $D_{1}^{s}$ was below $83 \%$. From previous studies (Dimitriadis et al., 2010; Harper et al., 2014), the low-frequency delta bands have also been noted by the research scientists as cognitive rhythms, and they associated these bands with attention and cognitive demanded tasks. Based on prediction results and previous studies, we decided to use $A_{4}^{s}$ and $D_{4}^{s}$ (delta low and delta high bands) for proposed system.

\section{Performance with Wavelet Coefficients Features}

To analyze the performance of the classifier, we used WCF features extracted at decomposition level-4. We performed the classification directly on the coefficients, instead of the features, which were obtained after wavelet decomposition at level 4 $\left(A_{4}\right.$ and $\left.D_{4}\right)$ by using three channels $\{f(t), p(t), c(t)\}$ with $2 \mathrm{D}$ and $3 \mathrm{D}$ contents. The first purpose of this analysis is to check the importance of these coefficients, whether these have discriminatory information or not. The second purpose of this analysis is to compare the accuracy results of WCF with the accuracy rate of the SWF. At DWT decomposition level 4, we obtained 27 coefficients (nine from each channel) from $A_{4}$ and $D_{4}$ (delta low and delta high sub-bands) separately. Then, we used the SVM classifier on these extracted coefficients by two ways, i.e., (i) with thresholding (WT) and (ii) without thresholding (WoT). The reason to apply a threshold on the extracted coefficients was to eliminate those coefficients that having very small values. In WT technique, we applied four different values of threshold, i.e., $\mathcal{E}=0.5,0.1,0.01,0.001$. In WoT technique, we applied the SVM classifier directly on the extracted coefficients without eliminating the small ones. Results are shown in Tables 3, 4 for $A_{4}$ and $D_{4}$ coefficients with $2 \mathrm{D}$ and $3 \mathrm{D}$ contents separately.

Figures 8, 9 show the accuracy of classifier against the coefficients extracted at wavelet decomposition level four for three channels with $2 \mathrm{D}$ and $3 \mathrm{D}$ contents. The best accuracy achieved by SVM classifier was 98.4 and $98.98 \%$ at $A_{4}$ and $D_{4}$ in $2 \mathrm{D}$ and $3 \mathrm{D}$, respectively. Figures 10, 11 show the AUC area under the receiver operating characteristic curve for $2 \mathrm{D}$ (HA vs. LA) and 3D (HA vs. LA) without thresholding.

\section{Performance with Statistical Wavelet Features}

To analyze the performance of classifier by using SWF features in various combinations, we used SVM with RBF kernel to classify

\begin{tabular}{|c|c|c|c|c|c|c|c|c|c|c|}
\hline \multirow[t]{3}{*}{ Epsilon } & \multicolumn{8}{|c|}{ With thresholding } & \multicolumn{2}{|c|}{ Without thresholding } \\
\hline & \multicolumn{2}{|c|}{0.5} & \multicolumn{2}{|c|}{0.1} & \multicolumn{2}{|c|}{0.01} & \multicolumn{2}{|c|}{0.001} & \multirow[b]{2}{*}{$\mathbf{A}_{4}$} & \multirow[b]{2}{*}{$\mathbf{D}_{4}$} \\
\hline & $\mathbf{A}_{4}$ & $\mathbf{D}_{4}$ & $A_{4}$ & $\mathbf{D}_{4}$ & $\mathbf{A}_{4}$ & $\mathbf{D}_{4}$ & $\mathbf{A}_{4}$ & $\mathbf{D}_{4}$ & & \\
\hline Accuracy (\%) & 82 & 90 & 88 & 90 & 86 & 84 & 88 & 94 & 97.5 & 97.9 \\
\hline Sensitivity (\%) & 79 & 89 & 87.2 & 86 & 84 & 83 & 86 & 93 & 98 & 98.60 \\
\hline Specificity (\%) & 77.20 & 86.8 & 85.1 & 84.6 & 82.4 & 80.2 & 83.3 & 92.8 & 96.8 & 97.10 \\
\hline Precision (\%) & 77.60 & 86.9 & 85.4 & 84.9 & 82.5 & 80.3 & 83.7 & 92.9 & 96.9 & 97.4 \\
\hline$A \cup C$ & 0.825 & 0.93 & 0.85 & 0.925 & 0.88 & 0.92 & 0.8 & 0.95 & 0.97 & 0.975 \\
\hline C & 8 & 2.83 & 8 & 8 & 11.3 & 2 & 2.83 & 8 & 2 & 2 \\
\hline$\gamma$ & 0.0014 & 0.004 & 0.002 & 0.002 & 0.0014 & 0.0055 & 0.002 & 0.0055 & 0.002 & 0.001 \\
\hline
\end{tabular}
the LA and HA classes with 2D and 3D contents separately. Our classification technique was used for all the features extracted

TABLE 3 | SVM Classifier Results (with RBF Kernel) in Classification of HA vs. LA (2D Content) with and without thresholding (for $A_{4}$ and $D_{4}$ coefficients), No. of channels $=03(F z, P z, C z)$. 
TABLE 4 | SVM Classifier Results (with RBF Kernel) in Classification of HA vs. LA (3D Content) with and without thresholding (for $A_{4}$ and $D_{4}$ coefficients), No. of channels $=03(F z, P z, C z)$.

\begin{tabular}{|c|c|c|c|c|c|c|c|c|c|c|}
\hline \multirow[t]{3}{*}{ Epsilon } & \multicolumn{8}{|c|}{ With thresholding } & \multicolumn{2}{|c|}{ Without thresholding } \\
\hline & \multicolumn{2}{|c|}{0.5} & \multicolumn{2}{|c|}{0.1} & \multicolumn{2}{|c|}{0.01} & \multicolumn{2}{|c|}{0.001} & \multirow[b]{2}{*}{$A_{4}$} & \multirow[b]{2}{*}{$\mathbf{D}_{4}$} \\
\hline & $A_{4}$ & $\mathrm{D}_{4}$ & $A_{4}$ & $\mathbf{D}_{4}$ & $A_{4}$ & $\mathrm{D}_{4}$ & $A_{4}$ & $\mathrm{D}_{4}$ & & \\
\hline Accuracy (\%) & 98 & 94 & 92 & 96 & 92 & 98 & 98 & 96 & 98 & 98.8 \\
\hline Sensitivity (\%) & 100 & 93.9 & 92.6 & 95.7 & 91.8 & 98.6 & 99 & 95.8 & 99.1 & 99.6 \\
\hline Specificity (\%) & 96.3 & 92.5 & 92.1 & 94.8 & 90.3 & 97.4 & 98.1 & 94.7 & 98.2 & 98.3 \\
\hline Precision (\%) & 96.4 & 92.7 & 92.4 & 94.9 & 90.8 & 97.5 & 98.6 & 94.8 & 98.3 & 98.5 \\
\hline$A \cup C$ & 0.975 & 0.94 & 0.89 & 0.97 & 0.95 & 0.97 & 0.97 & 0.96 & 0.975 & 0.98 \\
\hline C & 32 & 2 & 8 & 8 & 8 & 32 & 128 & 4 & 2 & 2 \\
\hline$\gamma$ & 0.003 & 0.0055 & 0.002 & 0.0055 & 0.0055 & 0.004 & 0.0007 & 0.004 & 0.0014 & 0.001 \\
\hline
\end{tabular}

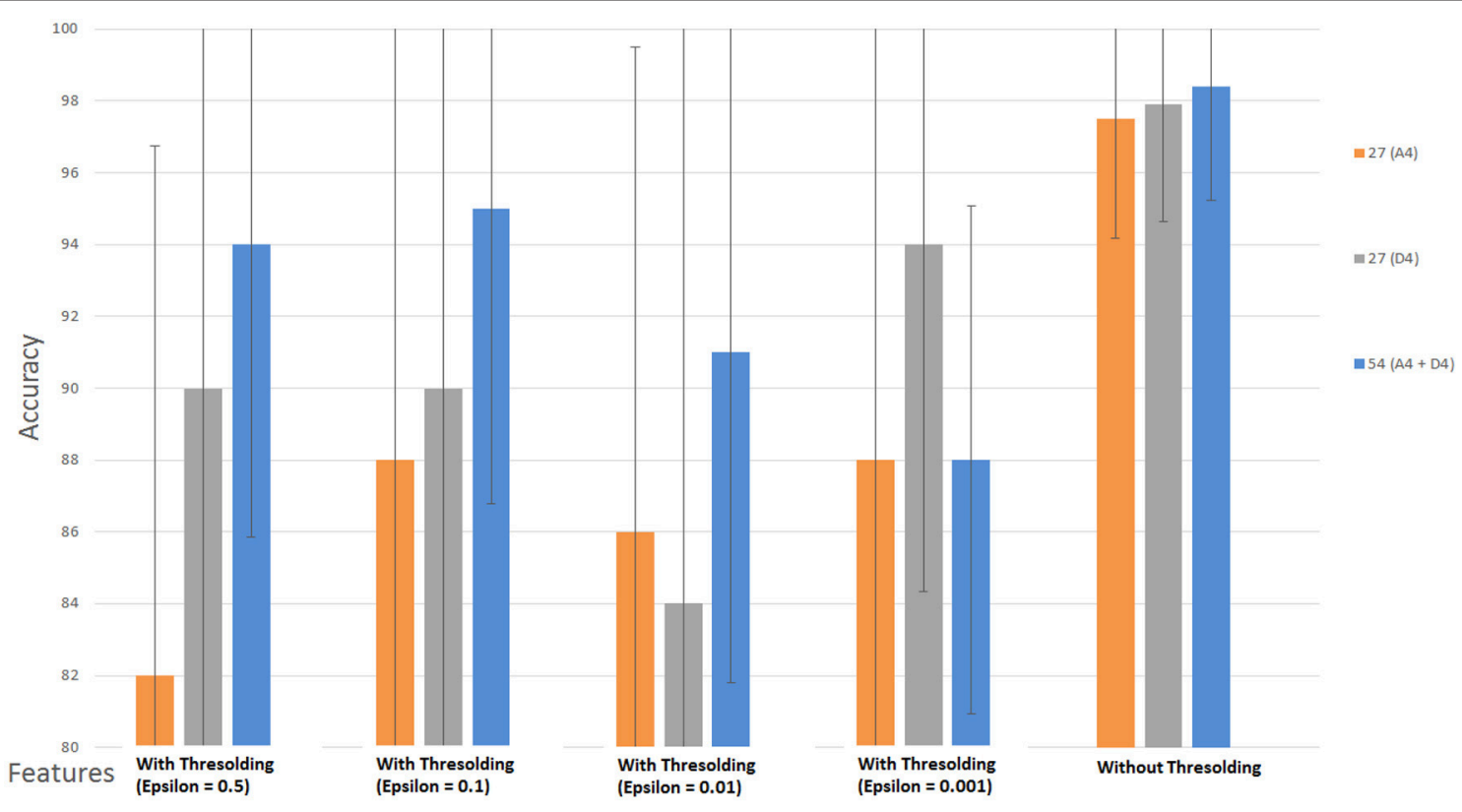

FIGURE 8 | Classification results of WCF features with and without thresholding using approximate (A4) and detailed coefficients (D4) for the cognitive task with 2D content (HA vs. LA). Wavelet type $=$ haar, level $=4$, No. of channels $=03(F z, P z, C z)$.

from all the sub-bands upto level 4 , i.e., $A_{4}$ and $D_{1}$ to $D_{4}$. However, the classifier results were not up to the mark for all the sub-bands. The classifier showed the best performance for two out of six extracted features, i.e., $E_{r}$ and $s t d$, each from the $A_{4}$ and $D_{4}$ coefficients at decomposition level 4 for both $2 \mathrm{D}$ and 3D contents. $A_{4}$ and $D_{4}$ represent the delta low and delta high band. The accuracy rate for the other bands, i.e., $D_{3}^{s}, D_{2}^{s}$ and $D_{1}^{s}$ was below $83 \%$. These results showed the domination of deltalow frequency $(0.0-1.875)$ and delta high frequency $(1.875-3.75$ $\mathrm{Hz}$ ) in the cognitive task. Results are shown in Tables 5-8 for $A_{4}$ and $D_{4}$ coefficients separately. In both $2 \mathrm{D}$ and $3 \mathrm{D}$ cases, best results were obtained for two features $\left(E_{r}\right.$ and $\left.s t d\right)$ for each of the three channels $\{f(t), p(t), c(t)\}$ of $X(t)$ for $A_{4}$ and $D_{4}$. Therefore, the total number of features was six (06) by using all three channels in 2D and 3D. By using these parameters, we obtained $100 \%$ accuracy in both 2D and 3D scenarios.
Figures 12, 13 show the accuracy of classifier against the different number of features for both $2 \mathrm{D}$ and $3 \mathrm{D}$ cases at wavelet decomposition level 4 . The best accuracy achieved by SVM classifier was $100 \%$ at $A_{4}$ and $D_{4}$ by using two features $\left(E_{r}\right.$ and $\left.s t d\right)$ for each channel in both $2 \mathrm{D}$ and $3 \mathrm{D}$ cases. Figures 14, 15 show the AUC area under the receiver operating characteristic curve for $2 \mathrm{D}$ (HA vs. LA) and $3 \mathrm{D}$ (HA vs. LA).

\section{Performance Using Different Subjects for Training and Testing Data}

In our evaluation so far, we used all trials together from all subjects and divided it into training and testing data using 10 -fold cross validation to test the performance of the system over different variations of the data. To show that the performance of the system does not depend on subjects, we also performed 


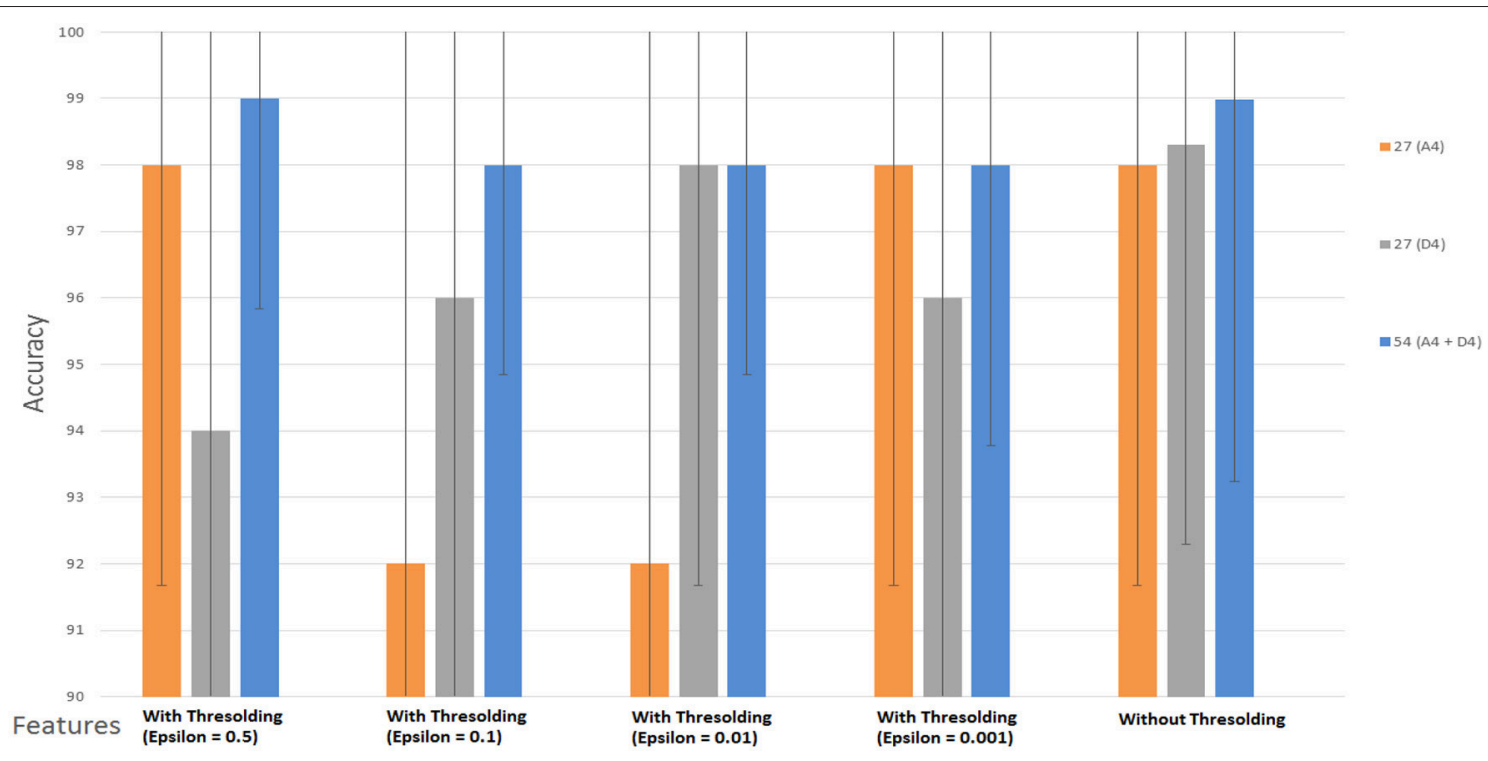

FIGURE 9 | Classification results of WCF features with and without thresholding using approximate (A4) and detailed coefficients (D4) for the cognitive task with 3D content (HA vs. LA). Wavelet type $=$ haar, level $=4$, No. of channels $=03$ ( $F z, P z, C z)$.

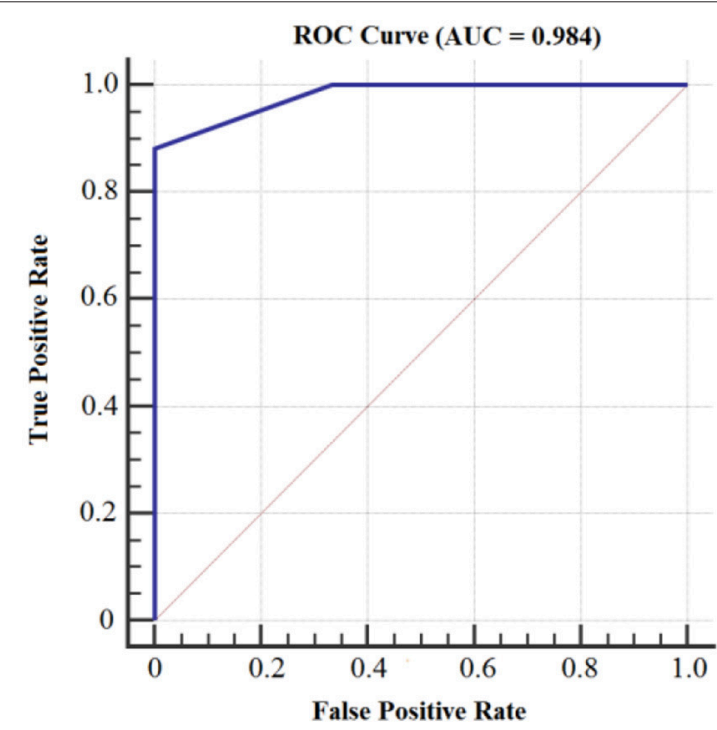

FIGURE 10 | AUC area under the receiver operating characteristic curve for 2D Content (HA vs. LA) without thresholding.

experiments by using testing data from the subjects, from whom the data was not used for training i.e., we used training and testing data from different subjects. For this purpose, we used the data from $70 \%$ of the subjects as training and the data from the remaining $30 \%$ of the subjects as testing, for both $2 \mathrm{D}$ and $3 \mathrm{D}$ cases. Using the features found to be the best in previous sections, i.e., $E_{r}$ and std of $A_{4}$ and $D_{4}$ sub-bands, the accuracy achieved by SVM classifier is $100 \%$ for both $2 \mathrm{D}$ and $3 \mathrm{D}$ cases. It indicated that the performance of the system is independent of subjects.

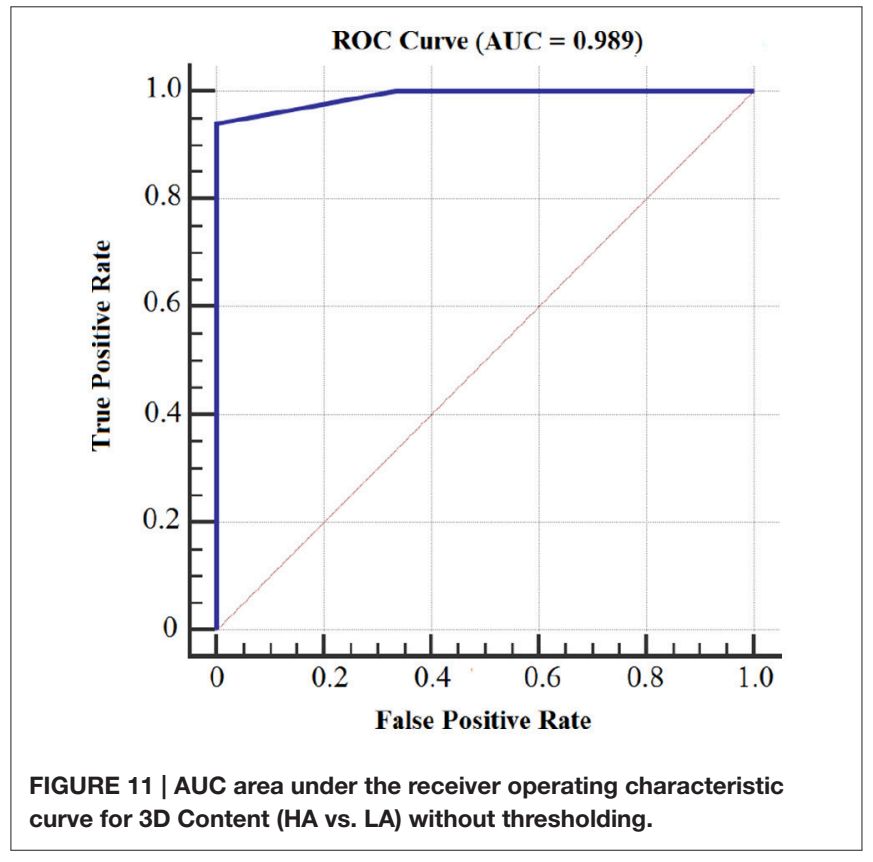

\section{Analysis of Discrimination of Features}

To analyze the discrimination of features giving the best performance, we used the following two different approaches:

(1) Statistical significance test,

(2) Inter-class and Intra-class variation analysis.

\section{Statistical Significance Test}

We used the statistical test to check the significance of our discriminatory SWF features. For this purpose, we performed 
TABLE 5 | SVM Classifier Results (with RBF Kernel) in Classification of HA vs. LA (2D Content) with SWF features for $A_{4}$ (delta low), No. of channels = 03 (Fz, Pz, Cz).

\begin{tabular}{|c|c|c|c|c|c|c|c|}
\hline No. of Features & Accuracy (\%) & Sensitivity (\%) & Specificity (\%) & Precision (\%) & AUC & C & $\gamma$ \\
\hline $6(E r, s t d)$ & 100 & 100 & 98.8 & 98.9 & 1 & 8 & 0.011 \\
\hline $9(E r, s t d, m)$ & 90 & 89 & 86.2 & 86.3 & 0.89 & 2048 & 0.00004 \\
\hline $9(k, s k, e)$ & 80 & 78.5 & 77.2 & 77.5 & 0.79 & 512 & 0.016 \\
\hline $12(E r, k, s k, e)$ & 90 & 88 & 87.7 & 87.9 & 0.89 & 512 & 0.016 \\
\hline 15(Er, std, k, sk, e) & 86.67 & 85 & 84 & 84.2 & 0.86 & 512 & 0.016 \\
\hline 18(Er, std, $m, k, s k, e)$ & 70 & 69 & 68.3 & 68.9 & 0.70 & 2048 & 0.0055 \\
\hline
\end{tabular}

Er, Energy; std, Standard Deviation; m, Mean; k, Kurtosis; sk, Skewness; e, Entropy.

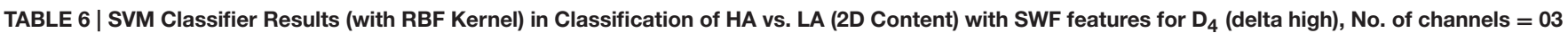
(Fz, Pz, Cz).

\begin{tabular}{|c|c|c|c|c|c|c|c|}
\hline No. of Features & Accuracy (\%) & Sensitivity (\%) & Specificity (\%) & Precision (\%) & AUC & C & $\gamma$ \\
\hline $6(E r, s t d)$ & 100 & 100 & 98.60 & 98.80 & 1.0 & 2 & 0.0014 \\
\hline $9(E r, s t d, m)$ & 90 & 91 & 88.10 & 88.20 & 0.88 & 2 & 0.0055 \\
\hline $9(k, s k, e)$ & 80 & 79 & 77.40 & 77.90 & 0.80 & 11.3 & 0.0014 \\
\hline 12(Er, k, sk, e) & 85 & 82 & 81.10 & 81.60 & 0.84 & 8192 & 0.0055 \\
\hline 15(Er, std, k, sk, e) & 80 & 78 & 77.30 & 77.60 & 0.79 & 42.3 & 0.0055 \\
\hline 18(Er, std, m, k, sk, e) & 73.33 & 71 & 70.70 & 70.90 & 0.73 & 2048 & 0.0052 \\
\hline
\end{tabular}

Er, Energy; std, Standard Deviation; m, Mean; k, Kurtosis; sk, Skewness; e, Entropy.

TABLE 7 | SVM Classifier Results (with RBF Kernel) in Classification of HA vs. LA (3D Content) with SWF features for $A_{4}$ (delta low), No. of channels = 03 $(F z, P z, C z)$.

\begin{tabular}{|c|c|c|c|c|c|c|c|}
\hline No. of Features & Accuracy (\%) & Sensitivity (\%) & Specificity (\%) & Precision (\%) & AUC & C & $\gamma$ \\
\hline $6(E r, s t d)$ & 100 & 100 & 98.20 & 98.40 & 1.0 & 2 & 0.0312 \\
\hline $9(E r, s t d, m)$ & 90 & 92 & 88.40 & 88.50 & 0.89 & 512 & 0.011 \\
\hline $9(k, s k, e)$ & 90 & 89 & 88.10 & 88.30 & 090 & 512 & 0.011 \\
\hline 12(Er, k, sk, e) & 90 & 89.10 & 88.50 & 88.60 & 0.89 & 512 & 0.011 \\
\hline 15(Er, std, k, sk, e) & 90 & 91.40 & 87.70 & 87.90 & 0.91 & 512 & 0.011 \\
\hline $18(E r, s t d, m, k, s k, e)$ & 76.66 & 76 & 75.20 & 75.50 & 0.76 & 512 & 0.016 \\
\hline
\end{tabular}

Er, Energy; std, Standard Deviation; m, Mean; k, Kurtosis; sk, Skewness; e, Entropy.

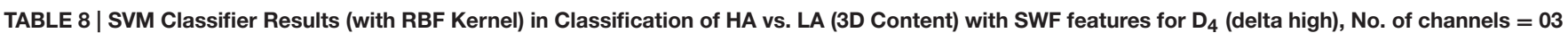
(Fz, Pz, Cz).

\begin{tabular}{|c|c|c|c|c|c|c|c|}
\hline No. of Features & Accuracy (\%) & Sensitivity (\%) & Specificity (\%) & Precision (\%) & AUC & C & $\gamma$ \\
\hline $6(E r, s t d)$ & 100 & 100 & 98.50 & 98.60 & 1 & 2 & 0.0005 \\
\hline $9(E r, s t d, m)$ & 90 & 89.10 & 88.30 & 88.60 & 0.88 & 512 & 0.011 \\
\hline $9(k, s k, e)$ & 90 & 91.20 & 88.70 & 88.80 & 0.89 & 8 & 0.0028 \\
\hline 12(Er, k, sk, e) & 80 & 80.50 & 78.10 & 78.30 & 0.79 & 32 & 0.0055 \\
\hline 15(Er, std, k, sk, e) & 90 & 89.70 & 87.60 & 87.90 & 0.898 & 42.3 & 0.0055 \\
\hline $18(E r, s t d, m, k, s k, e)$ & 66.67 & 66.50 & 64.20 & 64.50 & 0.66 & 11.3 & 0.0055 \\
\hline
\end{tabular}

Er, Energy; std, Standard Deviation; m, Mean; k, Kurtosis; sk, Skewness; e, Entropy.

the non-parametric procedure, i.e., Kruskal-Wallis Anova test. This test was basically used to compare the means between three or more independent groups. We applied this test in both $2 \mathrm{D}$ and $3 \mathrm{D}$ cases to check whether there is a significant difference between the features of LA and HA groups or not. For this purpose, we formed a hypothesis in $2 \mathrm{D}$ case, i.e., 


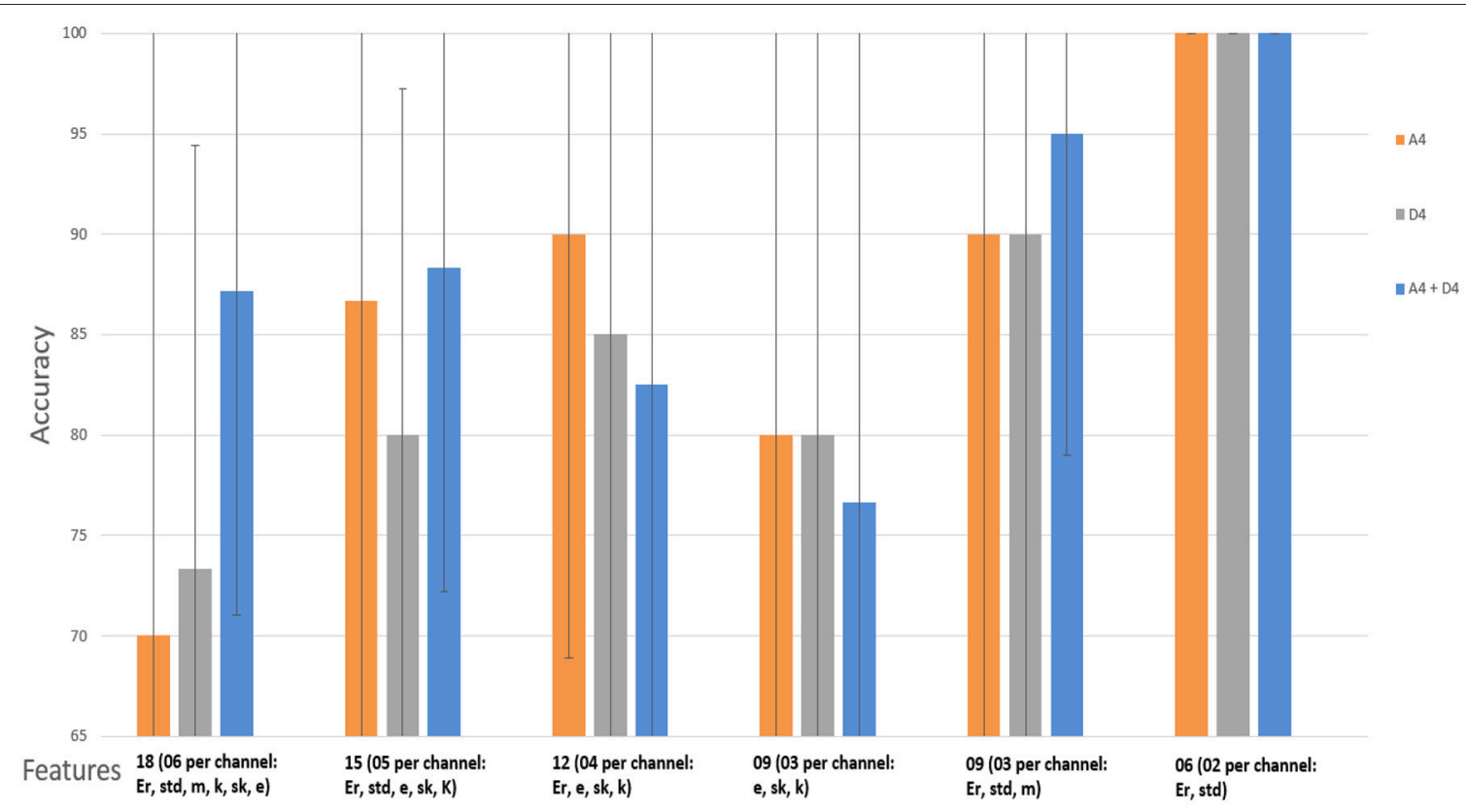

FIGURE 12 | Classification results of SWF features using approximate (A4) and detailed (D4) coefficients for the cognitive task with 2D content (HA vs. LA). Wavelet type $=$ haar, level = 4, No. of channels = 03 (Fz, Pz, Cz). Er, Energy; std, Standard Deviation; m, Mean; k, Kurtosis; sk, Skewness; e, Entropy.

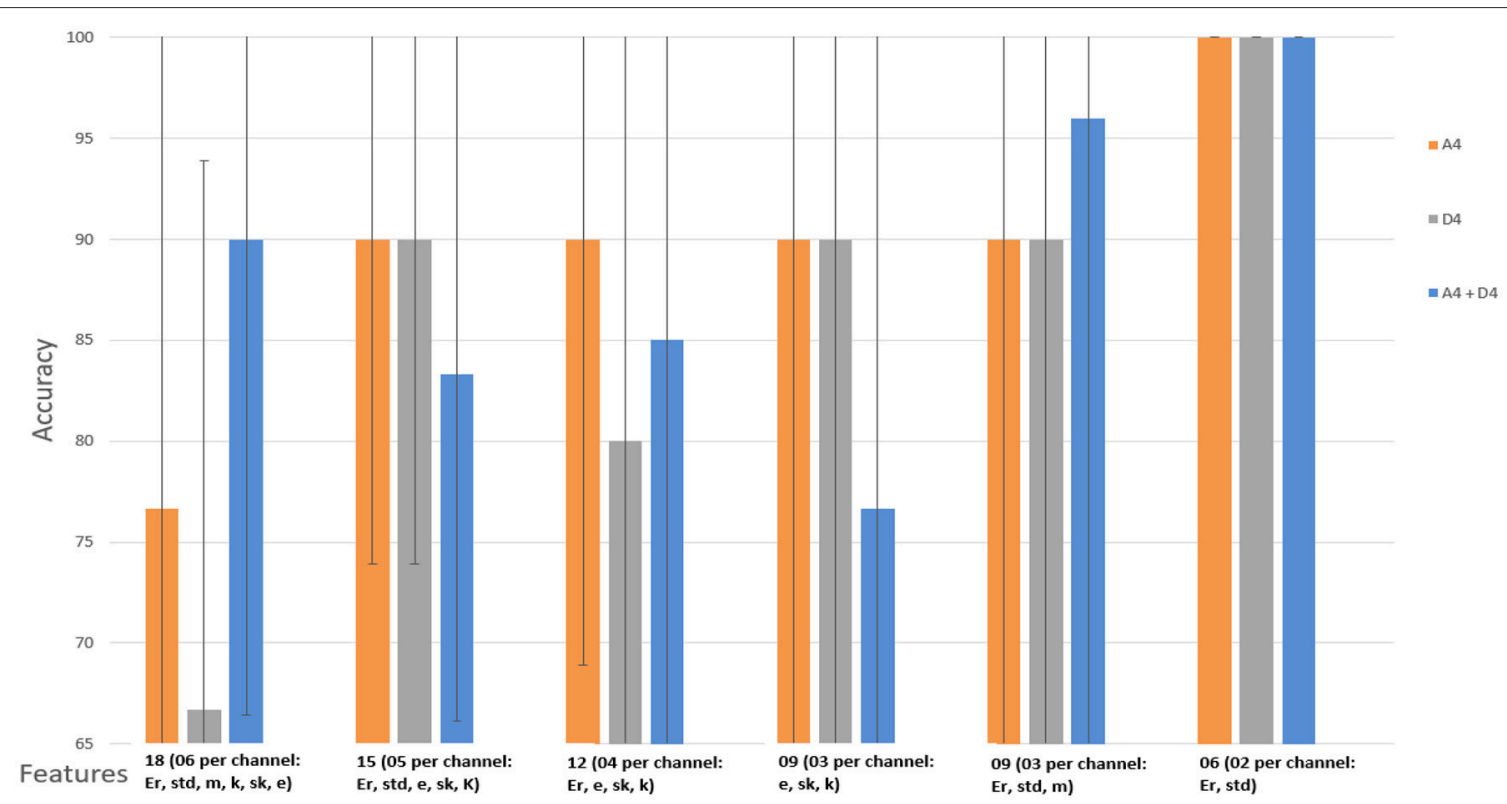

FIGURE 13 | Classification results of SWF features using approximate (A4) and detailed coefficients (D4) for the cognitive task with 3D content (HA vs. LA). Wavelet type = haar, level = 4, No. of channels = 03 (Fz, Pz, Cz). Er, Energy; std, Standard Deviation; m, Mean; k, Kurtosis; sk, Skewness; e, Entropy.

Null Hypothesis $\boldsymbol{H}_{0}$ : No significant difference exists between $\mathrm{HA}$ and LA classes in $2 \mathrm{D}$.

Alternate Hypothesis $\boldsymbol{H}_{1}$ : Significant difference exists between HA and LA classes in 2D.

We took three samples each from HA (sample \# 1 to 3 ) and LA classes (sample \# 4 to 6) from $E_{r}$ and std features, and applied
Kruskal-Wallis test on these samples. Figure 16 shows the result of Kruskal-Wallis test.

After performing the test, we got the $p=0$ which was less than alpha (0.05), therefore, we rejected the null hypothesis, and we can say that significant difference exists between HA and LA classes in 2D case. From Figure 16, we can also observe that first three samples are grouped together belonging to 


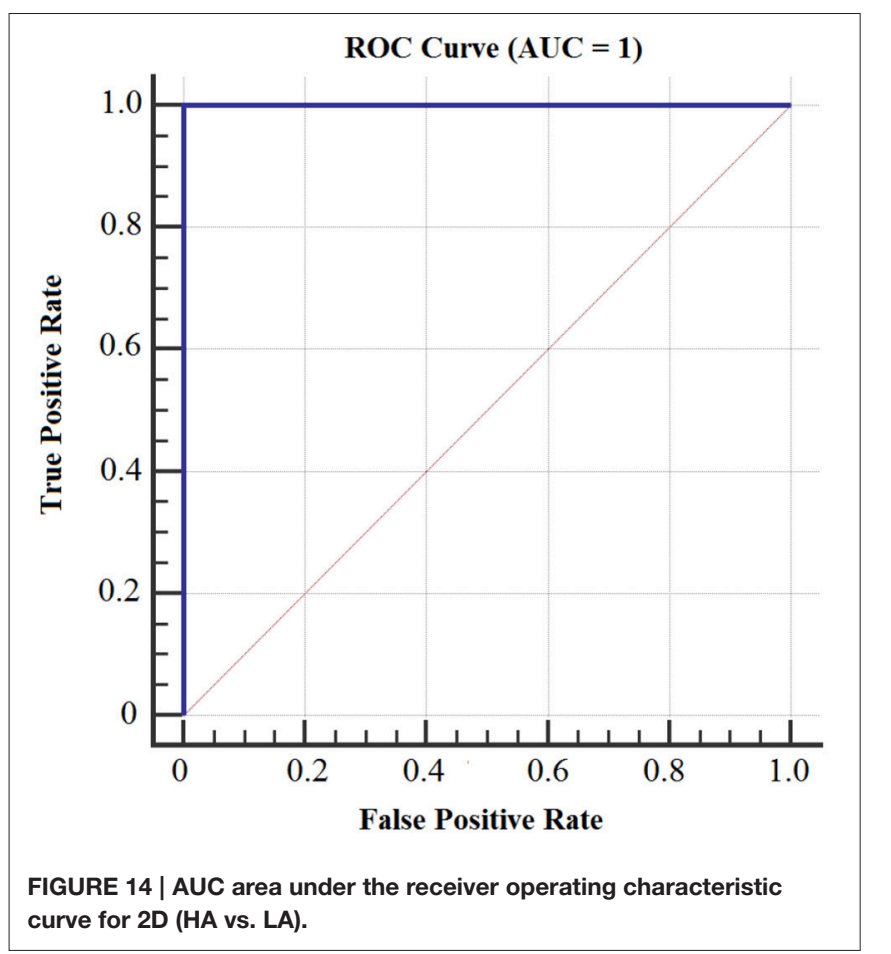

HA class (2D) and last three samples are grouped together belonging to LA class (2D). The samples of same class are close to each other; however, there is significant difference between the samples of different classes. Therefore, alternate hypothesis exists.

In $3 \mathrm{D}$ case, we also formed a hypothesis.

Null Hypothesis $\boldsymbol{H}_{0}$ : No significant difference exists between $\mathrm{HA}$ and LA classes in 3D.

Alternate $\mathbf{H y p o t h e s i s} \boldsymbol{H}_{1}$ : Significant difference exists between $\mathrm{HA}$ and LA classes in 3D.

Similarly, we took three samples each from the HA (sample \# 1 to 3 ) and LA classes (sample \# 4 to 6) from $E_{r}$ and std features and applied Kruskal-Wallis test on these samples. Figure 17 shows result of Kruskal-Wallis test.

After performing the test, we obtained the $p=2.3 \times$ $10^{-9}$ which was less than alpha $(0.05)$ so we rejected the null hypothesis, and we can say that significant difference exists between HA and LA classes in 3D case. From Figure 17, we can observe that first three samples are grouped together belonging to HA class (3D) and last three samples are grouped together belonging to LA class (3D). The samples of same class are close together; however, there is significant difference between the samples of different classes. Therefore, alternate hypothesis exists.

\section{Inter-Class and Intra-Class Variation Analysis}

We used the inter-class and intra-class variation analysis to show the significant difference between the SWF of LA and HA groups. For this analysis, we used the scatter matrices (Theodoridis and Koutroumbas, 2006) in 2D case. We calculated within-class


scatter matrix $\left(S_{w}\right)$ and between-class scatter matrix $\left(S_{b}\right)$ of LA and HA groups by taking the samples from the $E_{r}$ and std features. After getting the $S_{w}$ and $S_{b}$, we calculated the mixture scatter matrix $\left(S_{m}\right)$, i.e.,

$$
S_{m}=S_{w}+S_{b}
$$

Where $S_{m}$ represents the covariance matrix of the feature vector according to global mean. Then, we calculated the trace and determinant of $S_{m}$ and $S_{w}$. The criteria to check the significant difference between the features of two classes is that the trace $\left\{S_{m}\right\}$ should be greater than trace $\left\{S_{w}\right\}$, i.e., $J_{1}$, and determinant 


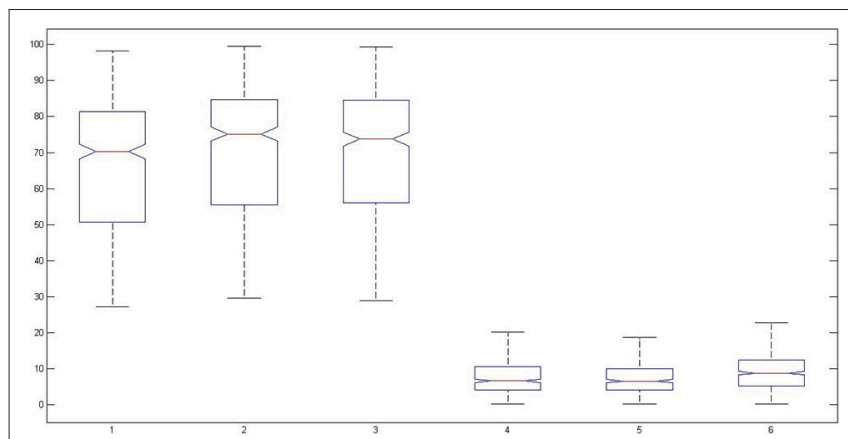

FIGURE 17 | Kruskal-Wallis Anova test for 3D case (HA vs. LA).



FIGURE 18 | Delta low $\left(A_{4}\right)$ in 0.0 to $1.875 \mathrm{~Hz}$ in HA (2D content).

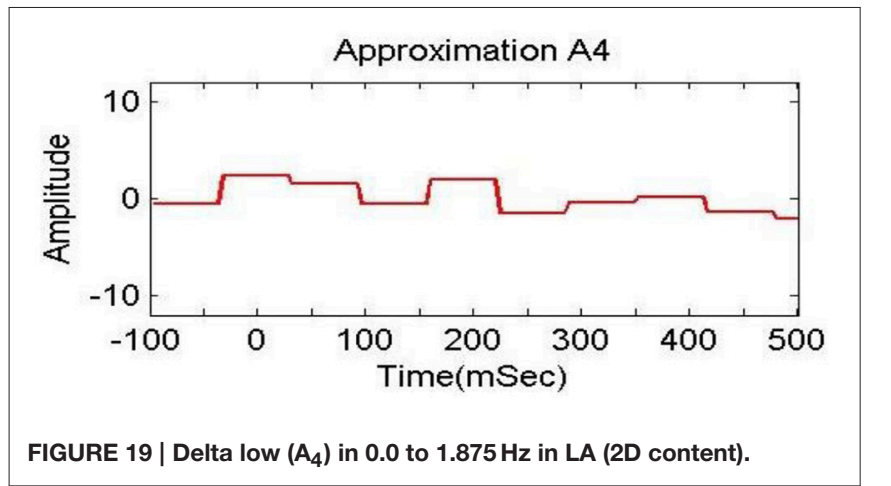

$\left\{S_{m}\right\}$ should also be greater than determinant $\left\{S_{w}\right\}$, i.e., $J_{2}$. It is represented as:

$$
\begin{aligned}
& J_{1}=\frac{\operatorname{trace}\left\{S_{m}\right\}}{\operatorname{trace}\left\{S_{w}\right\}} \\
& J_{2}=\frac{\operatorname{det}\left\{S_{m}\right\}}{\operatorname{det}\left\{S_{w}\right\}}
\end{aligned}
$$

It took larger values when samples in the L-dimensional space are well clustered around their mean, within each class and the clusters of the different classes are well separated. After using the Equations 16 and 17 , we got $J_{1}=11.0508$ and $J_{2}$ $=2.2570 \times 10^{3}$. This shows that criteria which define the condition, i.e., trace $\left\{S_{m}\right\}>$ trace $\left\{S_{w}\right\}$ and $\operatorname{det}\left\{S_{m}\right\}>\operatorname{det}$ $\left\{S_{w}\right\}$ holds. Therefore, analytical analysis shows that significant

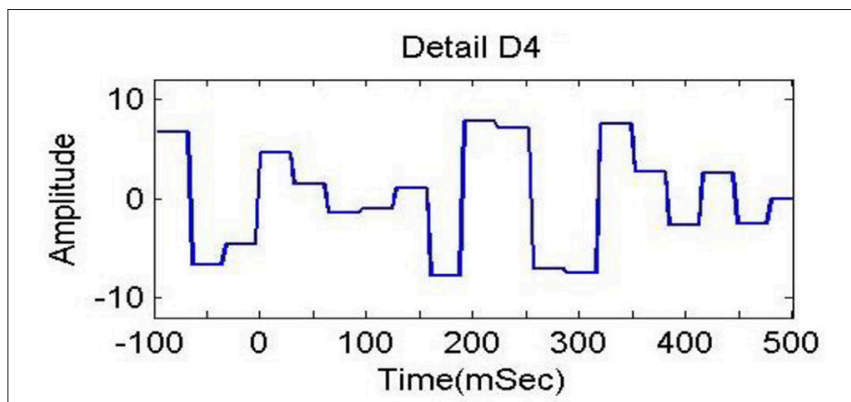

FIGURE 20 | Delta high $\left(D_{4}\right)$ in 1.875 to $3.75 \mathrm{~Hz}$ in HA (2D content).

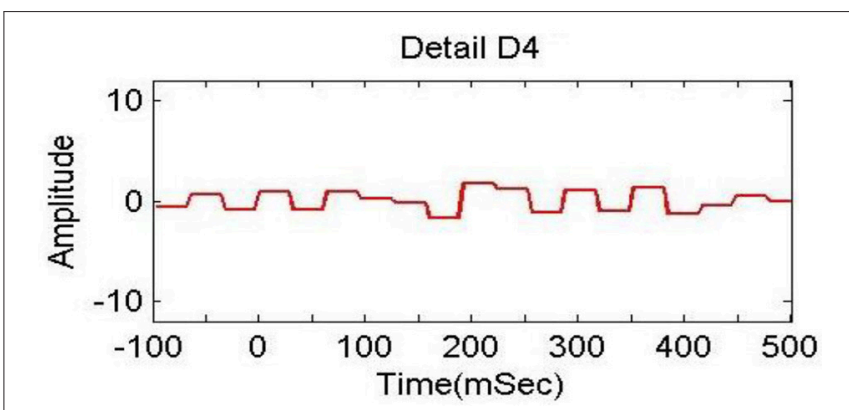

FIGURE 21 | Delta high $\left(D_{4}\right)$ in 1.875 to $3.75 \mathrm{~Hz}$ in LA (2D content).

difference exists between the features of LA and HA groups in $2 \mathrm{D}$ case.

\section{Analysis of EEG Signals}

To analyze the discrimination of EEG signals of LA and HA classes, we used different combinations of features in classification of SWF features, such as $6,9,12,15$, and 18 for our classification problem by utilizing all the three channels and both $A_{4}$ and $D_{4}$ coefficients at level 4 by considering 2D and $3 \mathrm{D}$ contents. We used different performance measuring parameters to check the result of our pattern recognition system like accuracy, sensitivity, specificity and precision. From the Tables 5-8, we can observe that SVM classifier got the 100\% accuracy rate in both $2 \mathrm{D}$ and $3 \mathrm{D}$ cases. Experimentally, we observed the classification rate by taking different number of features as mentioned in Figures 12, 13. We got the best classification rates, i.e., $100 \%$ for both $2 \mathrm{D}$ and $3 \mathrm{D}$ cases by using the two features, i.e., $E_{r}$ and $s t d$. These results show the discrimination between LA and HA classes by performing the cognitive task using the $2 \mathrm{D}$ and $3 \mathrm{D}$ content. A signal obtained from $\mathrm{Fz}$ channel by performing the cognitive task for the duration of $600 \mathrm{mS}$ in 2D case is shown in Figures 18-21. From the figures, we can analyze the clear difference between the amplitude of the signals in the delta low (0.0-1.875) and delta high (1.875-3.75) frequency bands for LA and HA classes in 2D case. These differences of amplitude verify that these frequency bands, i.e., delta low and delta high have discriminatory features $\left(E_{r}\right.$ and $\left.s t d\right)$ which help us to classify the LA and HA classes in 2D and $3 \mathrm{D}$ cases. 
In classification of WCF features with and without thresholding, the best accuracy rate achieved by SVM classifier was 98.4 and $98.98 \%$ at $\mathrm{A}_{4}$ and $\mathrm{D}_{4}$ in $2 \mathrm{D}$ and $3 \mathrm{D}$, respectively, as shown in Figures 8, 9. However, by applying the thresholding in $2 \mathrm{D}$ case, we can see in Figure 8 that its accuracy rate has been affected; however, without thresholding; its accuracy rate was much higher. It means that discriminatory information also exists in the small values of the coefficients. Therefore, all the coefficients extracted from the sub-bands at level-4 may be used for feature extraction in $2 \mathrm{D}$ case. In $3 \mathrm{D}$ case, we can see that from Figure 9, the accuracy rates have not been affected with and without thresholding. It gives almost the same accuracy in both cases. It means that elimination of coefficients in 3D case does not affect the accuracy rate. Therefore, we can use the coefficients obtained after the thresholding for the extraction of features.

In this study, we used three midline channels for the recording of EEG signals, i.e., $\mathrm{Fz}, \mathrm{Pz}, \mathrm{Cz}$. After getting the classification results as shown in Figures 12, 13, we can observe that these three channels play an important role in recording of signals during the cognitive task. From these signals, we extracted the discriminatory features to use in the classification problem. After achieving these results, we can conclude that the features, i.e., $E_{r}$ and $s t d$, which are extracted from the delta low (0.0 to $1.875 \mathrm{~Hz})$ and delta high $(1.875$ to $3.75 \mathrm{~Hz})$ frequency bands, can be used for the classification of EEG brain patterns recorded during the cognitive task.

\section{Analysis of Frequency Bands}

In this study, we extracted the two discriminatory features $\left(E_{r}\right.$ and $\left.s t d\right)$ from delta low and delta high bands, which were obviously low frequency bands, and then these features were used during the classification process. From $100 \%$ results that we achieved in this research study, we observed the importance of EEG low frequency bands in the classification problem. In Dimitriadis et al. (2010), Amin and Malik (2013), and Harper et al. (2014), the low-frequency EEG bands have also been noted by the research scientists as cognitive rhythms, and they associated these bands with attention and cognitive demanded tasks.

In ERP research studies (Ergen et al., 2008; Harper et al., 2014), researchers highlighted the importance of delta band by associating the $\mathrm{P} 300$ component with cognitive processes. In the neuroscience research literature, this association was extensively discussed. Delta band has also very important contribution toward the ERP's P300 Component (Demiralp et al., 1999). In another study (Gennady, 2012), authors confirmed the association between the delta band and cognitive processing. Some of the above-discussed studies showed the increase in the delta power during the cognitive tasks. Our research study also validates the findings of Amin et al. (2015a) to assess an individual's learning and memory recall ability based on P300 component. Due to above-mentioned reasons, we got the best accuracy rates during the classification process of EEG low frequency bands, i.e., delta low $(0.0-1.875 \mathrm{~Hz})$ and delta high $(1.875-3.75 \mathrm{~Hz})$ for discriminating the $\mathrm{HA}$ and LA ability groups in $2 \mathrm{D}$ and $3 \mathrm{D}$ cases by performing the visual oddball cognitive task.

\section{Effect of 2D and 3D Contents on High Ability and Low Ability Groups}

From the prediction accuracy, i.e., 100\%, we observed that there was no significant difference between the effect of $2 \mathrm{D}$ and $3 \mathrm{D}$ contents on HA and LA groups. By presenting both the contents, i.e., $2 \mathrm{D}$ and $3 \mathrm{D}$ to $\mathrm{HA}$ and LA groups, we achieved the same accuracy rate, i.e., 100\%. However, in order to evaluate the effect of $2 \mathrm{D}$ and $3 \mathrm{D}$ contents on HA and LA groups statistically, a hypothesis has been developed that measures the system performance related to $2 \mathrm{D}$ content whether it is less effective than $3 \mathrm{D}$ or not. For this purpose, we formed a hypothesis, i.e.,

Null Hypothesis $\boldsymbol{H}_{0}$ : No significant difference in the system performance related to $2 \mathrm{D}$ and $3 \mathrm{D}$ content.

Alternate Hypothesis $\boldsymbol{H}_{1}$ : System performance related to $2 \mathrm{D}$ content is less effective than $3 \mathrm{D}$.

In order to analyze the significant difference between $2 \mathrm{D}$ and $3 \mathrm{D}$ contents, we needed a large number of samples for accuracy values. Therefore, to meet this requirement, we run our system three times for 10 cross validation by utilizing the best parameters. Each time, we randomized our dataset. This process provides 30 prediction values for each system. In the next step, we utilized the independent t-test. After performing the $t$-test, we got $p=1$; which indicated that there is no significant difference exists in the system performance related to $2 \mathrm{D}$ and $3 \mathrm{D}$ contents. Null hypothesis was accepted as we got $p$-value greater than 0.05 . Therefore, we conclude statistically that there is no significant difference between the effect of $2 \mathrm{D}$ and $3 \mathrm{D}$ contents on HA and LA groups.

\section{Comparisons}

To evaluate the effectiveness of our proposed system, we compared our experimental results with previous EEG research studies. However, result comparisons with previous studies are complex due to various decomposition levels, wavelet types, variability of participants, and the variety of cognitive tasks. A detailed comparison of proposed methodology with previous research studies considering the performance of classifier, ML (machine learning) algorithm, cognitive tasks, dataset, and feature extraction methods is presented in Table 9. The research studies mentioned in Table 9 used the frequency domain, time domain, wavelet transform and autoregressive coefficients (AR) based features for the classification of EEG signals recorded during the cognitive task. Some of the research studies, mentioned in the comparison table, used the non-linear classifiers, are complex and more time-consuming to construct the classifier model. In research study (Subasi and Gursoy, 2010), authors used few instances only for the classification that results to create the overfitting issue. In this proposed methodology, we used a large number of trials or samples for each HA and LA classes, i.e., 551 for HA group (2D), 482 for LA group (2D), 433 for HA group (3D), and 529 for LA group (3D). We used a total number of 1995 trials. For each trial, we used six features (two from each channel) for the classification. We also used 10 -fold cross validation in the 
TABLE 9 | Comparison with existing techniques for EEG in cognitive tasks.

\begin{tabular}{|c|c|c|c|c|c|c|c|}
\hline Sr. no. & References & Subjects & $\begin{array}{c}\text { Scalp } \\
\text { electrodes }\end{array}$ & Feature & Classifier & Accuracy & Cognitive task \\
\hline 1 & Our Work & $\begin{array}{l}34 \text { (each for 2D and } \\
\text { 3D case separately) }\end{array}$ & 128 & $\begin{array}{l}\text { Relative Energy and } \\
\text { standard deviation }\end{array}$ & $\begin{array}{l}\text { SVM with RBF } \\
\text { Kernel }\end{array}$ & $\begin{array}{l}100 \% \text { (for both } \\
2 \mathrm{D} \text { and 3D) }\end{array}$ & $\begin{array}{l}\text { RAPM and Visual oddball } \\
\text { cognitive task }\end{array}$ \\
\hline 2 & Liang et al., 2006 & 07 & 6 & $\begin{array}{l}\text { Autoregressive } \\
\text { coefficients }\end{array}$ & $\begin{array}{l}\text { ELM, SVM and } \\
\text { ANN }\end{array}$ & $53.98-56.07$ & $\begin{array}{l}05 \text { tasks as mentioned in } \\
\text { Keirn and Aunon (1990) }\end{array}$ \\
\hline 3 & Diez et al., 2009 & 07 & 6 & $\begin{array}{l}\text { Frequency and time } \\
\text { domain features }\end{array}$ & LDA and ANN & $87.35-91.17$ & $\begin{array}{l}05 \text { tasks as mentioned in } \\
\text { Keirn and Aunon (1990) }\end{array}$ \\
\hline 4 & Lin and Hsieh, 2009 & 03 & 8 & Power feature & SVM and ANN & $65.90-68.35$ & $\begin{array}{l}03 \text { cognitive tasks (words } \\
\text { generation, imagination of left } \\
\text { and right hand movement) }\end{array}$ \\
\hline 5 & $\begin{array}{l}\text { Zhiwei and Minfen, } \\
2007\end{array}$ & 02 & 06 & $\begin{array}{l}\text { Wavelet packet } \\
\text { entropy }\end{array}$ & SVM & 87.5-93.0 & $\begin{array}{l}05 \text { tasks as mentioned in } \\
\text { Keirn and Aunon (1990) }\end{array}$ \\
\hline 6 & $\begin{array}{l}\text { Daud and Yunus, } \\
2004\end{array}$ & 04 & 06 & $\begin{array}{l}\text { Discrete wavelet } \\
\text { transform }\end{array}$ & ANN & $74.40-82.30$ & $\begin{array}{l}05 \text { tasks as mentioned in } \\
\text { Keirn and Aunon (1990) }\end{array}$ \\
\hline 7 & Hosni et al., 2007 & 07 & 06 & $\begin{array}{l}\text { Autoregressive } \\
\text { coefficients and } \\
\text { power of frequency } \\
\text { bands }\end{array}$ & SVM & 70 & $\begin{array}{l}\text { Three tasks (multiplication, } \\
\text { baseline and mental letter) }\end{array}$ \\
\hline 8 & Yazdani et al., 2009 & 04 & 06 & db4 wavelet & $\mathrm{K}-\mathrm{NN}$ & $81.48-89.58$ & $\begin{array}{l}05 \text { tasks as mentioned in } \\
\text { Keirn and Aunon (1990) }\end{array}$ \\
\hline 9 & Guo et al., 2011 & 07 & 06 & Immune feature & SVM & $85.40-97.5$ & $\begin{array}{l}05 \text { tasks as mentioned in } \\
\text { Keirn and Aunon (1990) }\end{array}$ \\
\hline 10 & Xue et al., 2003 & 04 & 06 & Wavelet packet & RBF network & 85.30 & $\begin{array}{l}05 \text { tasks as mentioned in } \\
\text { Keirn and Aunon (1990) }\end{array}$ \\
\hline
\end{tabular}

classification process. The advantage of 10 -fold cross validation scheme was that all the samples are used in the training and testing phase (Subasi and Gursoy, 2010). Therefore, by using a large number of trials or samples, we can compare our results with the previous studies to check the performance of classifier. From Table 9, we can observe that the results of our study are better than the previous studies, which are using the same or different classifier with the similar nature of the cognitive task.

\section{Limitations of the Study}

There are some limitations in the existing study, which will be considered in future research. In this study, all the individuals were male subjects. However, in future study, we will also consider female subjects in our experiment to predict the fluid intelligence level of both the genders. In future study, we will also consider some subjects that might have some medical problems, to analyze the effects of diseases on fluid intelligence level. There were only 34 subjects in the current study; therefore, number of subjects should be increased to prove that single trial EEG signals would be enough to predict the cognitive performance. Also, Raven's Advanced Progressive Matrices (RAPM) norm for university students of different nationalities will also be included in the future study. In addition, this research examined the association of EEG signals with memory and learning ability for young subjects only. In addition, the learning contents utilized in this research were associated to physiology and anatomy material; therefore, the results cannot be generalized to relate with learning capability of many other types of memory recall capability or academic learning materials. Finally, 128-chnanels, a very high-density EEG electrode system utilized in this research study cannot be considered appropriate for recording a few electrode EEG channels. In future study, we will utilize EEG recording devices with fewer electrodes according to the requirements of research.

\section{CONCLUSION}

In this research paper, we have proposed a system for predicting the fluid intelligence level of subjects, whether he/she belongs to LA and HA group using EEG single trial signals. For this purpose, we employed 2D and 3D contents and 34 subjects each for 2D and 3D, which were divided into low-ability (LA) and high-ability (HA) groups using RAPM test. Then, we used the visual oddball cognitive task to measure the neural activity of each group as three EEG signals $(F z, P z, C z)$. In order to predict whether a subject belongs to LA or HA group, we extracted two different types of Haar wavelet transform based features using wavelet decomposition from EEG signals in the band 0.3 to $30 \mathrm{~Hz}$. Then support vector machine (SVM) was used as a classifier with RBF kernel to classify the LA and HA groups in 2D and 3D cases. We achieved the 100\% classification rate in $2 \mathrm{D}$ and $3 \mathrm{D}$ cases with the domination of delta low $(0.0-1.875 \mathrm{~Hz})$ and delta high $(1.875-3.75 \mathrm{~Hz})$ frequency bands. After getting the best results, we conclude 
that $E_{r}$ and std are useful features in the classification of EEG brain patterns. In addition, the low-range frequency bands, i.e., delta band, show close association with the cognitive processes. The analysis of these frequency bands indicate clear difference between LA and HA groups. Furthermore, discriminative values of the features have been validated using statistical significance tests and inter-class and intra-class variation analysis. Also, statistical test shows that there is no effect of $2 \mathrm{D}$ and $3 \mathrm{D}$ content on fluid intelligence level. Comparison with state-of-theart techniques shows the significance of the proposed system. Therefore, our proposed system will be helpful in academic and clinical applications to predict the intelligence level of the subjects, and it may be implemented in online as well as offline applications.

\section{REFERENCES}

Acharya, U. R., Sree, S. V., Ang, P. C. A., Yanti, R., and Suri, J. S. (2012). Application of non-linear and wavelet based features for the automated identification of epileptic EEG signals. Int. J. Neural Syst. 22:1250002. doi: $10.1142 / S 0129065712500025$

Adeli, H., Zhou, Z., and Dadmehr, N. (2003). Analysis of EEG records in an epileptic patient using wavelet transform. J. Neurosci. Methods 123, 69-87. doi: 10.1016/S0165-0270(02)00340-0

Akay, M. (1997). Wavelet applications in medicine. IEEE Spectr. 34, 50-56. doi: $10.1109 / 6.590747$

Amin, H., and Malik, A. S. (2013). Human memory retention and recall processes: a review of EEG and fMRI studies. Neurosciences 18, 330-344.

Amin, H. U., Malik, A. S., Badruddin, N., and Chooi, W. T. (2013). "EEG mean power and complexity analysis during complex mental task," in ICME International Conference on Complex Medical Engineering (CME) 25-28 May. (Beijing).

Amin, H. U., Malik, A. S., Badruddin, N., Kamel, N., and Hussain, M. (2015b). "Effects of stereoscopic 3D display technology on event-related potentials (ERPs)," in 7th Annual International IEEE EMBS Conference on Neural Engineering (Montpellier), 22-24.

Amin, H. U., Malik, A. S., Kamel, N., Chooi, W. T., and Hussain, M. (2015a). P300 correlates with learning \& memory abilities and fluid intelligence. J. Neuroeng. Rehabil. 12, 87. doi: 10.1186/s12984-015-0077-6

Balas, B., and Koldewyn, K. (2013). Early visual ERP sensitivity to the species and animacy of faces. Neuropsychologia 51, 2876-2881. doi: 10.1016/j.neuropsychologia.2013.09.014

Daud, M., and Yunus, J. (2004). "Classification of mental tasks using denoised EEG signals," in 7th International Conference on Signal Processing (Beijing), 2206-2209.

Deary, I. J., Strand, S., Smith, P., and Fernandes, C. (2007). Intelligence and educational achievement. Intelligence 35, 13-21. doi: 10.1016/ j.intell.2006.02.001

deBettencourt, M., Goldman, R., Brown, T., and Sajda, P. (2011). Adaptive thresholding for improving sensitivity in single-trial simultaneous EEG/fMRI. Front. Psychol. 2:91. doi: 10.3389/fpsyg.2011.00091

Demiralp, T., Ademoglu, A., Schürmann, M., Basar-Eroglu, C., and Basar, E. (1999). Detection of P300 waves in single trials by the wavelet transform (WT). Brain Lang. 66, 108-128. doi: 10.1006/brln.1998.2027

Diez, P. F., Mut, V., Laciar, E., Torres, A., and Avila, E. (2009). "Application of the empirical mode decomposition to the extraction of features from EEG signals for mental task classfication," in Annual International Conference of the IEEE Engineering in Medicine and Biology Society, 2009, EMBC 2009 (Minnesota), 2579-2582.

Dimitriadis, S. I., Laskaris, N. A., Tsirka, V., Vourkas, M., and Micheloyannis, S. (2010). What does delta band tell us about cognitive processes: a mental calculation study. Neurosci. Lett. 483, 11-15. doi: 10.1016/j.neulet.2010.07.034

\section{AUTHOR CONTRIBUTIONS}

EQ and MH designed the study. EQ and HUA designed the experiment and collected the data. EQ developed the methodology. EQ, MH, HAS, AM, and SB performed the statistical analysis. EQ and $\mathrm{MH}$ performed the results interpretation and drafted the manuscript. All authors read and approved the final manuscript.

\section{ACKNOWLEDGMENTS}

This project was supported by NSTIP strategic technologies programs, grant number 12-INF2582-02 in the Kingdom of Saudi Arabia.

Ergen, M., Marbach, S., Brand, A., Başar-Eroğlu, C., and Demiralp, T. (2008). P3 and delta band responses in visual oddball paradigm in schizophrenia. Neurosci. Lett. 440, 304-308. doi: 10.1016/j.neulet.2008.05.054

Ferree, T. C. (2000). Spline Interpolation of the Scalp EEG. Technical Report, Secondary TitlEGI.

Garry, H., McGinley, B., Jones, E., and Glavin, M. (2013). An evaluation of the effects of wavelet coefficient quantisation in transform based EEG compression. Comput. Biol. Med. 43, 661-669. doi: 10.1016/j.compbiomed.2013.02.011

Gaspar, C. M., Rousselet, G. A., and Pernet, C. R. (2011). Reliability of ERP and single-trial analyses. NeuroImage 58, 620-629. doi: 10.1016/j.neuroimage.2011. 06.052

Gennady, G. K. (2012). EEG delta oscillations as a correlate of basic homeostatic and motivational processes. Neurosci. Biobehav. Rev. 36, 677-695. doi: 10.1016/j.neubiorev.2011.10.002

Goldman, R. I., Wei, C. Y., Philiastides, M. G., Gerson, A. D., Friedman, D., Brown, T. R., et al. (2009). Single-trial discrimination for integrating simultaneous EEG and fMRI: identifying cortical areas contributing to trialto-trial variability in the auditory oddball task. Neuroimage 47, 136-147. doi: 10.1016/j.neuroimage.2009.03.062

Gonzalez, R. C., and Woods, R. E. (2002). Digital Image Processing, 2nd Edn. Upper Saddle River, NJ: Prentice Hall.

Gray, J. R., Chabris, C. F., Braver, T. S. (2003). Neural mechanisms of general fluid intelligence. Nat. Neurosci. 6, 316-322. doi: 10.1038/nn1014

Guo, L., Wu, Y., Zhao, L., Cao, T., Yan, W., and Shen, X. (2011). Classification of mental task from EEG signals using immune feature weighted support vector machines. IEEE Trans. Magn. 47, 866-869. doi: 10.1109/TMAG.2010.2072775

Hariharan, M., Vijean, V., Sindhu, R., Divakar, P., Saidatul, A., and Yaacob, S. (2014). Classification of mental tasks using stockwell transform. Comput. Electr. Eng. 40, 1741. doi: 10.1016/j.compeleceng.2014.01.010

Harper, J., Malone, S. M., and Bernat, E. M. (2014). Theta and delta band activity explain N2 and P3 ERP component activity in a go/no-go task. Clin. Neurophysiol. 125, 124-132. doi: 10.1016/j.clinph.2013.06.025

Hosni, S. M., Gadallah, M. E., Bahgat, S. F., and AbdelWahab, M. S. (2007). "Classification of EEG signals using different feature extraction techniques for mental-task BCI," in International Conference on Computer Engineering and Systems, 2007, ICCES'07 (Cairo), 220-226.

Huettel, S. A., and McCarthy, G. (2004). What is odd in the oddball task?: prefrontal cortex is activated by dynamic changes in response strategy. Neuropsychologia 42, 379-386. doi: 10.1016/j.neuropsychologia.2003.07.009

Hussain, M., Wajid, S. K., Elzaart, A., and Berbar, M. (2011). "A comparison of SVM kernel functions for breast cancer detection," in Eighth International Conference Computer Graphics, Imaging and Visualization (Singapore).

Iscan, Z., Dokur, Z., and Demiralp, T. (2011). Classification of electroencephalogram signals with combined time and frequency features. Expert Syst. Appl. 38, 10499-10505. doi: 10.1016/j.eswa.2011.02.110

Jahankhani, P., Kodogiannis, V., and Revett, K. (2006). "EEG signal classification using wavelet feature extraction and neural networks," in IEEE John Vincent 
Atanasoff 2006 International Symposium on Modern Computing, 2006, JVA'06 (Los Alamitos, CA), 120-124.

Jahidin, A., Ali, M. M., Taib, M. N., Tahir, N. M., Yassin, I. M., and Lias, S. (2014). Classification of intelligence quotient via brainwave subband power ratio features and artificial neural network. Comput. Methods Programs Biomed. 114, 50-59. doi: 10.1016/j.cmpb.2014.01.016

Jongsma, M. L., Gerrits, N. J., van Rijn, C. M., Quiroga, R. Q., and Maes, J. H. (2012). Event related potentials to digit learning: tracking neurophysiologic changes accompanying recall performance. Int. J. Psychophysiol. 85, 41-48. doi: 10.1016/j.ijpsycho.2011.10.004

Karkare, S., Saha, G., and Bhattacharya, J. (2009). Investigating longrange correlation properties in EEG during complex cognitive tasks. Chaos Solitons Fractals 42, 2067-2073. doi: 10.1016/j.chaos.2009.03.148

Keirn, Z. A., and Aunon, J. I. (1990). A new mode of communication between man and his surroundings. IEEE Trans. Biomed. Eng. 37, 1209-1214. doi: $10.1109 / 10.64464$

Liang, N.-Y., Saratchandran, P., Huang, G.-B., and Sundararajan, N. (2006). Classification of mental tasks from EEG signals using extreme learning machine. Int. J. Neural Syst. 16, 29-38. doi: 10.1142/S0129065706000482

Lin, C.-J., and Hsieh, M.-H. (2009). Classification of mental task from EEG data using neural networks based on particle swarm optimization. Neurocomputing 72, 1121-1130. doi: 10.1016/j.neucom.2008.02.017

Nai-Jen, H., and Palaniappan, R. (2004). "Classification of mental tasks using fixed and adaptive autoregressive models of EEG signals," in 26th Annual International Conference of the IEEE Engineering in Medicine and Biology Society, 2004, IEMBS'04 (San Francisco, CA), 507-510.

Ocak, H. (2008). Optimal classification of epileptic seizures in EEG using wavelet analysis and genetic algorithm. Signal. Process. 88, 1858-1867. doi: 10.1016/j.sigpro.2008.01.026

Orhan, U., Hekim, M., and Ozer, M. (2011). EEG signals classification using the K-means clustering and a multilayer perceptron neural network model. Expert Syst. Appl. 38, 13475-13481. doi: 10.1016/j.eswa.2011. 04.149

Polich, J. (2007). Updating P300: an integrative theory of P3a and P3b. Clin. Neurophysiol. 118, 2128-2148. doi: 10.1016/j.clinph.2007.04.019

Primi, R., Ferrão, M. E., and Almeida, L. S. (2010). Fluid intelligence as a predictor of learning: a longitudinal multilevel approach applied to math. Learn. Individ. Differ. 20, 446-451. doi: 10.1016/j.lindif.2010.05.001

Quiroga, R. Q., Atienza, M., Cantero, J. L., and Jongsma, M. L. A. (2007). What can we learn from single-trial event-related potentials? Chaos Complexity Lett. 2, 345-363.

Ratcliff, R., Philiastides, M. G., and Sajda, P. (2009). Quality of evidence for perceptual decision making is indexed by trial-to-trial variability of the EEG. Proc. Natl. Acad. Sci. U.S.A. 106, 6539-6544. doi: 10.1073/pnas.0812 589106

Raven, J. (2000). The Raven's progressive matrices: change and stability over culture and time. Cogn. Psychol. 41, 1-48. doi: 10.1006/cogp.1999.0735

Ricardo, P., Eugenia, M. F., and Leandro, S. (2010). Almeida. Fluid intelligence as a predictor of learning: a longitudinal multilevel approach applied to math. Learn. Individ. Differ. 20, 446-451. doi: 10.1016/j.lindif.2010.05.001

Richman, J. S., and Moorman, J. R. (2000). Physiological time-series analysis using approximate entropy and sample entropy. Am. J. Physiol. Heart Circ. Physiol. 278, H2039-H2049.

Rodrıguez-Bermudez, G., Garcia-Laencina, P. J., Roca-Gonzalez, J., and RocaDorda, J. (2013). Efficient feature selection and linear discrimination of EEG signals. Neurocomputing 115, 161-165. doi: 10.1016/j.neucom.2013.01.001

Rosso, O. A., Blanco, S., Yordanova, J., Kolev, V., Figliola, A., Schürmann, M., et al. (2001). Wavelet entropy: a new tool for analysis of short duration brain electrical signals. J. Neurosci. Methods 105, 65-75. doi: 10.1016/S0165-0270(00)00356-3

Rousselet, G. A., Gaspar, C. M., Wieczorek, K. P., and Pernet, C. R. (2011). Modeling single-trial ERP reveals modulation of bottom-up face visual processing by top-down task constraints (in some subjects). Front. Psychol. 2:137. doi: 10.3389/fpsyg.2011.00137

Schneider, W., Eschman, A., and Zuccolotto, A. (2002). Book E-Prime User's Guide. Pittsburgh, PA: Psychology Software Tools Inc.
Schyns, P. G. (2010). Grand challenges in perception science: modeling the future. Front. Psychol. 1:10. doi: 10.3389/fpsyg.2010.00010

Soltani, S. (2002). On the use of the wavelet decomposition for time series prediction. Neurocomputing 48, 267-277. doi: 10.1016/S0925-2312(01)00648-8

Stollnitz, E. J., DeRose, T. D., and Salesin, D. H. (1995). Wavelets for computer graphics: a primer, part 1. IEEE Comput. Graph. 15, 76-84. doi: $10.1109 / 38.376616$

Subasi, A. (2007). EEG signal classification using wavelet feature extraction and a mixture of expert model. Expert Syst. 32, 1084-1093. doi: 10.1016/j.eswa.2006.02.005

Subasi, A., and Gursoy, M. I. (2010). EEG signal classification using PCA, ICA, LDA, and support vector machines. Expert Syst. 37, 8659-8666. doi: 10.1016/j.eswa.2010.06.065

Teplan, M. (2002). Fundamentals of EEG measurement. Meas. Sci. Rev. 2, 1-11.

Thatcher, R. W., North, D., and Biver, C. (2005). EEG and intelligence: relations between EEG coherence, EEG phase delay and power. Clin. Neurophysiol. 116, 2129-2141. doi: 10.1016/j.clinph.2005.04.026

Theodoridis, S., and Koutroumbas, K. (2006). Pattern Recognition, 3rd Edn. St. Louis, MO: Elsevier.

Theodoridis, S., and Koutroumbas, K. (2009). "Chapter 5-feature selection," in Pattern Recognition 4thEdn., eds S. Theodoridis and K. Koutroumbas (Boston, MA: Academic Press), 261-322.

Ubeyli, E. D. (2009). Combined neural network model employing wavelet coefficients for EEG signals classification. Digit. Signal. Proc. 19, 297-308. doi: 10.1016/j.dsp.2008.07.004

Ubeyli, E. D. (2010). Lyapunov exponents/probabilistic neural networks for analysis of EEG signals. Expert Syst. Appl. 37, 985-992. doi: 10.1016/j.eswa. 2009.05.078

Van den Bos, W., Crone, E. A., and Guroglu, B. (2012). Brain function during probabilistic learning in relation to IQ and level of education. Dev. Cogn. Neurosci. 2, S78-S89. doi: 10.1016/j.den.2011.09.007

Vidaurre, C., Kramer, N., Blankertz, B., and Schlogl, A. (2009). Time domain parameters as a feature for EEG-based brain-computer interfaces. Neural Netw. 22, 1313-1319. doi: 10.1016/j.neunet.2009.07.020

Wang, T., Ren, X., Altmeyer, M., and Schweizer, K. (2013). An account of the relationship between fluid intelligence and complex learning in considering storage capacity and executive attention. Intelligence $41,537-545$. doi: 10.1016/j.intell.2013.07.008

Wronka, E., Kaiser, J., and Coenen, A. M. L. (2013). Psychometric intelligence and P3 of the event-related potentials studied with a 3-stimulus auditory oddball task. Neurosci. Lett. 535, 110-115. doi: 10.1016/j.neulet.2012.12.012

Xue, J.-Z., Zhang, H., Zheng, C.-X., and Yan, X.-G. (2003). "Wavelet packet transform for feature extraction of EEG during mental tasks," in International Conference on Machine Learning and Cybernetics, 2003 (Xi'an), 360-363.

Yazdani, A., Ebrahimi, T., and Hoffmann, U. (2009). "Classification of EEG signals using Dempster Shafer theory and a k-nearest neighbor classifier," in 4th International IEEE/EMBS Conference on Neural Engineering (Antalya), $327-330$

Zhang, L., He, W., He, C., and Wang, P. (2010). Improving mental task classification by adding high frequency band information. J. Med. Syst. 34, 51-60. doi: 10.1007/s10916-008-9215-z

Zhiwei, L., and Minfen, S. (2007). "Classification of mental task EEG signals using wavelet packet entropy and SVM," in 8th International Conference on Electronic Measurement and Instruments, 2007, ICEMI'07 (Xi'an), 3-906-3-909.

Conflict of Interest Statement: The authors declare that the research was conducted in the absence of any commercial or financial relationships that could be construed as a potential conflict of interest.

Copyright (c) 2017 Qazi, Hussain, Aboalsamh, Malik, Amin and Bamatraf. This is an open-access article distributed under the terms of the Creative Commons Attribution License (CC BY). The use, distribution or reproduction in other forums is permitted, provided the original author(s) or licensor are credited and that the original publication in this journal is cited, in accordance with accepted academic practice. No use, distribution or reproduction is permitted which does not comply with these terms. 